\title{
Complexity Theoretic Limitations on Learning Halfspaces
}

\author{
Amit Daniely*
}

March 15, 2016

\begin{abstract}
We study the problem of agnostically learning halfspaces which is defined by a fixed but unknown distribution $\mathcal{D}$ on $\mathbb{Q}^{n} \times\{ \pm 1\}$. We define $\operatorname{Err}_{\mathrm{HALF}}(\mathcal{D})$ as the least error of a halfspace classifier for $\mathcal{D}$. A learner who can access $\mathcal{D}$ has to return a hypothesis whose error is small compared to $\operatorname{Err}_{\mathrm{HALF}}(\mathcal{D})$.

Using the recently developed method of [33] we prove hardness of learning results assuming that random $K$-XOR formulas are hard to (strongly) refute. We show that no efficient learning algorithm has non-trivial worst-case performance even under the guarantees that $\operatorname{Err}_{\mathrm{HALF}}(\mathcal{D}) \leq$ $\eta$ for arbitrarily small constant $\eta>0$, and that $\mathcal{D}$ is supported in $\{ \pm 1\}^{n} \times\{ \pm 1\}$. Namely, even under these favorable conditions, and for every $c>0$, it is hard to return a hypothesis with error $\leq \frac{1}{2}-\frac{1}{n^{c}}$. In particular, no efficient algorithm can achieve a constant approximation ratio. Under a stronger version of the assumption (where $K$ can be poly-logarithmic in $n$ ), we can take $\eta=2^{-\log ^{1-\nu}(n)}$ for arbitrarily small $\nu>0$. These results substantially improve on previously known results $[31,38,50,51,44]$, that only show hardness of exact learning.
\end{abstract}

* Department of Mathematics, The Hebrew University and Microsoft Research Herzliya. 


\section{Introduction}

In the problem of agnostically learning halfspaces, a learner is given an access to a distribution $\mathcal{D}$ on $\mathbb{Q}^{n} \times\{ \pm 1\}$. The goal is to output ${ }^{1}$ (a description of) a classifier $h: \mathbb{Q}^{n} \rightarrow\{ \pm 1\}$ whose error, $\operatorname{Err}_{\mathcal{D}}(h):=\operatorname{Pr}_{(x, y) \sim \mathcal{D}}(h(x) \neq y)$, is small comparing to $\operatorname{Err}_{\text {HALF }}(\mathcal{D})$ - the error of the best classifier of the from $h_{w}(x)=\operatorname{sign}(\langle w, x\rangle)$. We say that a learning algorithm learns halfspaces if, given an accuracy parameter $\epsilon>0$, it outputs a classifier with error at most $\operatorname{Err}_{\mathrm{HALF}}(\mathcal{D})+\epsilon$. The learner is efficient if it runs in time poly $\left(n, \frac{1}{\epsilon}\right)$ and the output hypothesis can be evaluated in poly $\left(n, \frac{1}{\epsilon}\right)$ time given its description. The learner has an approximation ratio $\alpha=\alpha(n)$ if it is guaranteed to return $h$ with $\operatorname{Err}_{\mathcal{D}}(h) \leq \alpha \cdot \operatorname{Err}_{\mathrm{HALF}}(\mathcal{D})+\epsilon$. We emphasize that we consider the general, improper, setting where the learner has the freedom to return a hypothesis that is not a halfspace classifier.

The problem of learning halfspaces is as old as the field of machine learning, starting with the perceptron algorithm [56, 55], through the modern SVM [27, 61, 60, 28, 29] and AdaBoost [58, 57, 40]. Halfspaces are widely used in practice, have been extensively studied theoretically, and in fact motivated much of the existing theory, both the statistical and the computational.

Despite all that, the gap between the performance of best known algorithms and best known lower bounds is dramatic. Best known efficient algorithms [48] for the problem have a poor approximation ratio of $\tilde{\Omega}(n)$, and have performance better than trivial only when $\operatorname{Err}_{\mathrm{HALF}}(\mathcal{D}) \leq \tilde{\Theta}\left(\frac{1}{n}\right)$. As for lower bounds, strong $\mathcal{N} \mathcal{P}$-hardness results are known $[8,5,41,38]$ only for algorithms that are restricted to return a halfspace classifier (a.k.a. proper algorithms). For general algorithms, no $\mathcal{N} \mathcal{P}$-hardness results are known, yet several results $[31,38,50,51,44]$ show that it is hard to agonstically learn halfspaces under several (cryptographic and average case) complexity assumptions. However, these results are quantitatively very weak, as they only rule out exact learning (i.e., with approximation ratio 1). For example, they do not rule out algorithms that predict only 1.001 times worst than the best halfspace classifier.

The main result of this paper is a quantitatively strong hardness results, assuming that (strongly) refuting random $K$-XOR formulas is hard. Using the recently developed framework of the author with Linial and Shalev-Shwartz [33], we show that for arbitrarily small constant $\eta>0$ and every $c>0$, no $\operatorname{poly}(n)$-time algorithm can return a hypothesis with error $\leq \frac{1}{2}-\frac{1}{n^{c}}$, even when it is guaranteed that $\operatorname{Err}_{\mathrm{HALF}}(\mathcal{D}) \leq \eta$, and that $\mathcal{D}$ is supported in $\{ \pm 1\}^{n} \times\{ \pm 1\}$. This implies in particular, that there is no efficient learning algorithm with a constant approximation ratio. Under a stronger version of the assumption (where $K$ is allowed to be poly-logarithmic in $n$ ), we can take $\eta=2^{-\log ^{1-\nu}(n)}$ for arbitrarily small $\nu>0$. This implies hardness of approximation up to a factor of $2^{\log ^{1-\nu}(n)}$. Interestingly, this is even stronger than the best known results [8, 41, 38] for proper algorithms.

\subsection{The random $K$-XOR assumption}

Unless we face a dramatic breakthrough in complexity theory, it seems unlikely that hardness of learning can be established on standard complexity assumptions such as $\mathbf{P} \neq \mathbf{N P}$ (see [6, 33]). Indeed, all currently known lower bounds are based on cryptographic and average-case assumptions. One type of such assumptions, on which we rely in this paper, concern the random $K$-XOR problem. This problem has been extensively studied, and assumptions about its intractability were used to prove hardness of approximation results [1], establish public-key cryptography [1, 7], and statisticalcomputational tradeoffs [11].

\footnotetext{
${ }^{1}$ Throughout, we require algorithms to succeed with a constant probability (that can be standardly amplified by repetition).
} 
A $K$-tuple is a mapping $C:\{ \pm 1\}^{n} \rightarrow\{ \pm 1\}^{K}$ in which each output coordinate is a literal and the $K$ literals correspond to $K$ different variables. The collection of $K$-tuples is denoted $\mathcal{X}_{n, K}$. A $K$-formula is a collection $J=\left\{C_{1}, \ldots, C_{m}\right\}$ of $K$-tuples. An instance to the $K$-XOR problem is a $K$-formula, and the goal is to find an assignment $\psi \in\{ \pm 1\}^{n}$ that maximizes $\operatorname{VAL}_{\psi, \mathrm{XOR}}(J):=$ $\frac{\left|\left\{j: \mathrm{XOR}_{K}\left(C_{i}(\psi)\right)=1\right\}\right|}{m}$. We define the value of $J$ as $\operatorname{VAL}_{\mathrm{XOR}}(J):=\max _{\psi \in\{ \pm 1\}^{n}} \operatorname{VAL}_{\psi, \mathrm{XOR}}(J)$. We will allow $K$ to vary with $n$ (but still be fixed for every $n$ ). For example, we can look of the $\lceil\log (n)\rceil$-XOR problem.

We will consider the problem of distinguishing random formulas from formulas with high value. Concretely, for $m=m(n), K=K(n)$ and $\frac{1}{2}>\eta=\eta(n)>0$, we say that the problem $\mathrm{CSP}_{m}^{\text {rand, } 1-\eta}\left(\mathrm{XOR}_{K}\right)$ is easy, if there exists an efficient randomized algorithm, $\mathcal{A}$ with the following properties. Its input is a $K$-formula $J$ with $n$ variables and $m$ constraints and its output satisfies:

- If $\operatorname{VAL}_{\mathrm{XOR}}(J) \geq 1-\eta$, then

$$
\operatorname{Pr}_{\text {coins of } \mathcal{A}}(\mathcal{A}(J)=\text { "non-random" }) \geq \frac{3}{4}
$$

- If $J$ is random ${ }^{2}$, then with probability $1-o_{n}(1)$ over the choice of $J$,

$$
\operatorname{Pr}_{\text {coins of } \mathcal{A}}(\mathcal{A}(J)=\text { "random" }) \geq \frac{3}{4} .
$$

It is not hard to see that the problem gets easier as $m$ gets larger, and as $\eta$ gets smaller. For $\eta=0$, the problem is actually easy, as it can be solved using Gaussian elimination. However, even for slightly larger $\eta$ 's the problems seems to become hard. For example, for any constant $\eta>0$, best known algorithms $[37,25,26,11,3]$ only work with $m=\Omega\left(n^{\frac{K}{2}}\right)$. In light of that, we put forward the following two assumptions.

Assumption 1.1 There are constants $c>0$ and $\frac{1}{2}>\eta>0$ such that for every $K$ and $m=$ $n^{c \log (K) \sqrt{K}}$, the problem $\mathrm{CSP}_{m}^{\mathrm{rand}, 1-\eta}\left(\mathrm{XOR}_{K}\right)$ is hard.

Assumption 1.2 There are constants $c>0$ and $\frac{1}{2}>\eta>0$ such that for every $s, K=\log ^{s}(n)$ and $m=n^{c K}$, the problem $\mathrm{CSP}_{m}^{\mathrm{rand}, 1-\eta}\left(\mathrm{XOR}_{K}\right)$ is hard.

We outline below some evidence to the assumptions, in addition to known algorithms' performance.

Hardness of approximation. Hastad's celebrated result [43] asserts that if $\mathbf{P} \neq \mathbf{N P}$, then for every $\eta>0$, it is hard to distinguish $K$-XOR instances with value $\geq 1-\eta$ from instances with with value $\leq \frac{1}{2}+\eta$. Since the value of a random formula is approximately $\frac{1}{2}$, we can interpret Hastad's result as claiming that it is hard to distinguish formulas with value $1-\eta$ from "semi-random" $K$-XOR formulas (i.e., formulas whose value is approximately the value of a random formula). Therefore, our assumptions can be seen as a strengthening of Hastad's result.

Hierarchies and SOS lower bounds. A family of algorithms whose performance has been analyzed are convex relaxations $[24,59,2]$ that belong to certain hierarchies of convex relaxations. Among those hierarchies, the strongest is the Lasserre hierarchy (a.k.a. Sum of Squares). Algorithms from this family achieves state of the art results for the $K$-XOR and many similar problems. In [59] it is shown that relaxations in the Lasserre hierarchy that work in sub-exponential time

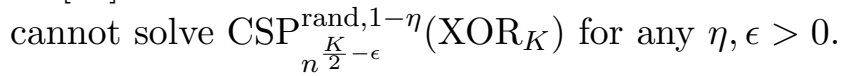

\footnotetext{
${ }^{2}$ To be precise, the $K$-tuples are chosen uniformly, and independently from one another.
} 
Lower bounds on statistical algorithms. Another family of algorithms whose performance has been analyzed are the so-called statistical algorithms. Similarly to hierarchies lower bounds, the results in [39] imply that statistical algorithms cannot solve $\operatorname{CSP}_{n^{\frac{K}{2}-\epsilon}}^{\mathrm{rand}, 1-\eta}\left(\mathrm{XOR}_{K}\right)$ for any $\eta, \epsilon>0$.

Resolution lower bounds. The length of resolution refutations of random $K$-XOR formulas have been extensively studied (e.g. [42, 13, 14, 19]). It is known (Theorem 2.24 in [18]) that random formulas with $n^{\frac{K}{2}-\epsilon}$ constraints only have exponentially long resolution refutations. This shows that yet another large family of algorithms (the so-called Davis-Putnam algorithms [35]) cannot efficiently solve $\mathrm{CSP}_{n^{\frac{K}{2}-\epsilon}}^{\mathrm{rand}, 1}\left(\mathrm{XOR}_{K}\right)$ for any $\epsilon>0$.

Similar assumptions. Several papers relied on similar assumptions. Alekhnovich [1] assumed that $\operatorname{CSP}_{C n}^{\text {rand,1- } 1}\left(\mathrm{XOR}_{3}\right)$ is hard for some $\eta<\frac{1}{2}$ and for every $C>0$. Applebaum, Barak and Wigderson [7] assumed that $\mathrm{CSP}_{n^{1.4}}^{\mathrm{rand}, 1-n^{-0.2}}\left(\mathrm{XOR}_{3}\right)$ is hard. Barak and Moitra [11] made the assumption that $\mathrm{CSP}_{n^{\frac{K}{2}-\epsilon}}^{\mathrm{rand}, 1-\eta}\left(\mathrm{XOR}_{K}\right)$ is hard for every $\epsilon>0$ and $\eta=\frac{1}{2}-o(1)$. Assumptions on predicates different than $K$-XOR were made in $[32,36]$. The assumption in [36] implies that $\mathrm{CSP}_{C n}^{\mathrm{rand}, 1-\eta}\left(\mathrm{XOR}_{3}\right)$ is hard for every $C>0$ and $\eta>0$. The assumption in [32] implies that

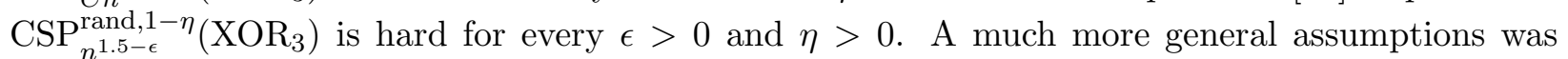
made in [12]. It implies in particular that $\mathrm{CSP}_{C n}^{\mathrm{rand}, 1-\eta}\left(\mathrm{XOR}_{K}\right)$ is hard for every $C>0$ and $\eta>0$.

\subsection{Previous Results and Related work}

Upper bounds. When $\operatorname{Err}_{\text {HALF }}(\mathcal{D})=0$, the problem of learning halfspaces can be solved efficiently using linear programming. However, even for slightly larger error values the problem seems to become much harder. Currently best known algorithms [48] have non trivial performance only when it is guaranteed that $\operatorname{Err}_{\mathrm{HALF}}(\mathcal{D}) \leq \frac{\log (n)}{n}$. This algorithm also achieves approximation ratio of $n$, which is currently the best known approximation ratio. Better guarantees are known under various assumptions on the marginal distribution [10, 20, 30, 44]. For example, a PTAS is known [30] when the marginal distribution is uniform.

Lower bounds for general algorithms. Several hardness assumptions imply that it is hard to agnostically learn halfspaces. Feldman, Gopalan, Khot and Ponnuswami [38] have shown that based on the security of the Ajtai-Dwork cryptosystem. Kalai, Klivans, Mansour and Servedio showed the same conclusion [44] based on the hardness of learning parity with noise. Daniely and Shalev-Shwartz [31] derived the same conclusion based on the hardness of refuting random $K$-SAT formulas. Klivans and Kothari [50] showed that assuming that learning sparse parity is hard, it is hard to learn halfspaces even when the marginal distribution is Gaussian. We note that all these results only rule out exact algorithms, but say nothing about approximation algorithms. We note however that by $[9,15]$, an algorithm with non trivial performance of $\eta$-realizable distributions will result with quasi-polynomial time algorithm for learning constant depth circuits.

Lower bounds for proper algorithms and hardness of approximation. When we restrict the algorithms to return a halfspace classifier, the problem of learning halfspaces is essentially equivalent [54] to the computational problem of minimizing disagreements. In this problem we are given a sample $S=\left\{\left(x_{1}, y_{1}\right), \ldots,\left(x_{m}, y_{m}\right)\right\} \in \mathbb{Q}^{n} \times\{ \pm 1\}$, and the goal is to find a vector $w \in \mathbb{Q}^{n}$ that minimizes the fraction of pairs with $\operatorname{sign}\left(\left\langle w, x_{i}\right\rangle\right) \neq y_{i}$. The optimal fraction is called the error of the sample. As a "standard" and basic computational problem, much is known about it. The problem have been shown $\mathcal{N} \mathcal{P}$-hard already in Karp's famous paper [46]. Soon after the discovery of the PCP theorem, Arora, Babai, Stern and Sweedyk [8] have shown that assuming that 
no quasi-polynomial time algorithm can solve $\mathcal{N} \mathcal{P}$-hard problems, there is no efficient algorithm with an approximation ratio of $2^{\log ^{1-\epsilon}(n)}$. Later on, the corresponding maximization problem was considered by several authors [4, 17, 23, 41, 38], culminating with Feldman, Gopalan, Khot and Ponnuswami [38] who showed that assuming that no quasi-polynomial time algorithm can solve $\mathcal{N P}$ hard problems, no efficient algorithm can distinguish samples with error $\geq \frac{1}{2}-2^{-\sqrt{\log (n)}}$ from samples with error $\leq 2^{-\sqrt{\log (n)}}$.

Statistical-Queries Lower bounds. Statistical queries (SQ) algorithms [47] is a class of learning algorithms whose interaction with $\mathcal{D}$ is done only via statistical queries. Concretely, an SQalgorithm can specify any function $Q:\{ \pm 1\}^{n} \times\{ \pm 1\} \rightarrow\{ \pm 1\}$ and an error parameter $\lambda>0$, and receive a number $e$ satisfying $\left|E_{(x, y) \sim \mathcal{D}}[Q(x, y)]-e\right| \leq \lambda$. In this model, an algorithm is efficient if it makes polynomially many queries with error parameters satisfying $\frac{1}{\lambda} \leq \operatorname{poly}(n)$ (besides that, the algorithm is not restricted). While this class is strictly smaller than the class of all algorithms, most known algorithms admit an SQ version. In addition, as opposed to general algorithms, unconditional lower bounds are known for SQ algorithms [21] for several learning problems. In particular, it is known that it is hard to agnostically learn halfspaces using SQ-algorithm (e.g. [45]). We note that as with previously known lower bounds for general algorithms, these results only rule out exact algorithms.

Lower bounds on concrete algorithms. A few results [16, 53, 34] showing hardness of approximation results for several concrete families of algorithms (linear methods).

The methodology of [33]. In light of the great success of complexity theory in establishing hardness of approximation results for standard computational problems, having such dramatic gaps between upper and lower bounds is perhaps surprising. The reason for the discrepancy between learning problems and computational problems is the fact that it is unclear how to reduce NP-hard problems to learning problems (see $[6,33]$ ). The main obstacle is the ability of a learning algorithm to return a hypothesis which does not belong to the learnt class (in our case, halfspaces). Until recently, there was only a single framework, due to Kearns and Valiant [49], to prove lower bounds on learning problems. The framework of [49] makes it possible to show that certain cryptographic assumptions imply hardness of certain learning problems. As indicated above, the lower bounds established by this method are quite far from the performance of best known algorithms.

In a recent paper [33] (see also [32]) we, together with Linial and Shalev-Shwartz, developed a new framework to prove hardness of learning based on hardness on average of CSP problems. Yet, in [33] we were not able to use our technique to establish hardness results that are based on a natural assumption on a well studied problem. Rather, we made a rather speculative hardness assumption, that is concerned with general CSP problems, most of which were never studied explicitly. We recognized it as the main weakness of our approach, and therefore the main direction for further research. About a year after, Allen, O'Donnell and Witmer [3] refuted the assumption of [33]. On the other hand we [31] were able to overcome the use of our speculative assumption, and prove hardness of learning of DNF formulas (and other problems) based on a natural assumption on the complexity of refuting random $K$-SAT instances, in the spirit of Feige's assumption [36]. The current paper continues this line of work. 


\subsection{Results}

Main result We say that a distribution $\mathcal{D}$ on $\mathbb{Q}^{n} \times\{ \pm 1\}$ is $\eta$-almost realizable if $\operatorname{Err}_{\text {HALF }}(\mathcal{D}) \leq \eta$. An algorithm have non-trivial performance w.r.t. a certain family of distributions if for some $c>0$, its output hypothesis has error $\leq \frac{1}{2}-\frac{1}{n^{c}}$ whenever the underlying distribution belongs to the family.

Theorem 1.3 - Under assumption 1.1, for all $\eta>0$, there is no poly $(n)$-time algorithm with non-trivial performance on $\eta$-almost-realizable distributions on $\{ \pm 1\}^{n} \times\{ \pm 1\}$.

- Under assumption 1.2, for all $\nu>0$, there is no poly(n)-time algorithm with non-trivial performance on $2^{-\log ^{1-\nu}(n)}$-almost-realizable distributions on $\{ \pm 1\}^{n} \times\{ \pm 1\}$.

These results imply in particular that under assumption 1.1 there is no efficient learning algorithm with a constant approximation ratio, and under assumption 1.2 the is no efficient learning algorithm with an approximation ratio of $2^{\log ^{1-\nu}(n)}$. As mentioned above, this substantially improves on previously known results that only showed hardness of exact learning.

Extension to large margin learning Large margin learning is a popular variant of halfspace learning (e.g. [60,61]). Here, the learning algorithm faces an somewhat easier task, as it is not required to classify correctly examples that are very close to the separating hyperplane. Our basic theorem can be extended to the large margin case. Concretely, we say that a distribution $\mathcal{D}$ on $\{ \pm 1\}^{n} \times\{ \pm 1\}$ is $\eta$-almost-realizable-with-margin if there is $w \in \mathbb{R}^{d}$ with $\sum_{i=1}^{n}\left|w_{i}\right| \leq \operatorname{poly}(n)$ such that $\operatorname{Pr}_{(x, y) \sim \mathcal{D}}(y \cdot\langle w, x\rangle \leq 1) \leq \eta$. Theorem 1.3 can be extended to show that no efficient algorithm can perform better than trivial even when the distribution is almost realized with margin. Concretely, we have the following theorem.

Theorem 1.4 - Under assumption 1.1, for all $\eta>0$, there is no poly $(n)$-time algorithm with non-trivial performance on distributions on $\{ \pm 1\}^{n} \times\{ \pm 1\}$ that are $\eta$-almost-realizable-withmargin.

- Under assumption 1.2, for all $\nu>0$, there is no poly(n)-time algorithm with non-trivial performance on distributions on $\{ \pm 1\}^{n} \times\{ \pm 1\}$ that are $2^{-\log ^{1-\nu}(n)}$-almost-realizable-withmargin.

Statistical queries version Our proof technique can be adapted to show unconditional lower bounds for SQ-algorithms. Concretely, we have the following.

Theorem 1.5 There is no efficient SQ-algorithm with non-trivial performance on distributions on $\{ \pm 1\}^{n} \times\{ \pm 1\}$ that are $2^{-\log ^{1-\nu}(n)}$-almost-realizable-with-margin.

Again, this substantially improves on previously known results that only showed hardness of exact learning.

Implications to hardness of approximation As explained in the previous section, the problem of proper learning is essentially equivalent to the problem of minimizing disagreements. As our results hold in particular for proper algorithms, we can conclude the following.

Theorem 1.6 Under assumption 1.2, for every $\epsilon>0$ and $c>0$, no efficient algorithm can distinguish samples with error $\geq \frac{1}{2}-n^{-c}$ from samples with error $\leq 2^{-\log ^{1-\epsilon}(n)}$. 
We note that the conclusion of our theorem is stronger than the conclusions of the previously best known lower bounds for the problem (however, we rely on a stronger assumption). In particular, it implies that the problem is hard to approximate within a factor of $2^{\log ^{1-\epsilon}(n)}$, implying the conclusion [8] of Arora et. al. It is also strengthen the conclusion [38] of Feldman et. al., improving the completeness parameter from $2^{-\sqrt{\log (n)}}$ to $2^{-\log ^{1-\epsilon}(n)}$ and the soundness parameter from $\frac{1}{2}-$ $2^{-\sqrt{\log (n)}}$ to $\frac{1}{2}-n^{-c}$. We remark that our result holds even if we assume that the input examples are binary, and in the large margin settings.

\section{Main proof ideas}

We next elaborate on the main ideas of our main theorem. The full proof is deferred to the appendix.

\subsection{The methodology of [33]}

We first describe the methodology of [33] to prove hardness of learning. A sample is a collection $S=\left\{\left(x_{1}, y_{1}\right), \ldots,\left(x_{m}, y_{m}\right)\right\} \subset X \times\{ \pm 1\}$. The error of $h: X \rightarrow\{ \pm 1\} \operatorname{is}_{\operatorname{Err}_{h}}(S)=\frac{\left|\left\{j: h\left(x_{j}\right) \neq y_{j}\right\}\right|}{m}$. The error of $S$ w.r.t. a hypothesis class $\mathcal{H} \subset\{ \pm 1\}^{X}$ is $\operatorname{Err}_{\mathcal{H}}(S)=\min _{h \in \mathcal{H}} \operatorname{Err}_{h}(S)$. The basic idea behind [33] is that if it is hard to distinguish a sample with small error form a sample that is very random in a certain sense, then it is hard to (even approximately) learn $\mathcal{H}$.

For the sake of concreteness, we restrict to the problem of learning halfspaces over the boolean cube. Namely, we take $X=\{ \pm 1\}^{n}$ and $\mathcal{H}=\mathrm{HALF}=\left\{h_{w} \mid w \in \mathbb{R}^{n}\right\}$. We say that a sample is strongly scattered ${ }^{3}$ if the labels (i.e., the $y_{i}$ 's) are independent fair coins (in particular, they are independent from the $x_{i}$ 's). For $m=m(n)$ and $\eta=\eta(m)$, we denote by $\mathrm{HALF}_{m}^{\mathrm{s}-\mathrm{scat}, \eta}$ the problem of distinguishing a strongly-scattered sample from a sample with $\operatorname{Err}_{\mathrm{HALF}}(S) \leq \eta$. Concretely, we say that the problem is easy if there exists an efficient randomized algorithm $\mathcal{A}$ with the following properties. Its input is a sample $S=\left\{\left(x_{1}, y_{1}\right), \ldots,\left(x_{m}, y_{m}\right)\right\} \subset\{ \pm 1\}^{n} \times\{ \pm 1\}$, and its output satisfies:

- If $\operatorname{Err}_{\mathrm{HALF}}(S) \leq \eta$, then

$$
\operatorname{Pr}_{\text {coins of } \mathcal{A}}(\mathcal{A}(S)=\text { "almost-realizable" }) \geq \frac{3}{4}
$$

- If $S$ is strongly scattered then, with probability $1-o_{n}(1)$ over the choice of the labels,

$$
\operatorname{Pr}_{\text {coins of } \mathcal{A}}\left(\mathcal{A}(S)=\text { "scattered") } \geq \frac{3}{4} .\right.
$$

Theorem 2.1 [33] If for every $a>0$ the problem $\mathrm{HALF}_{n^{a}}^{\mathrm{s}-\mathrm{scat}, \eta}$ is hard, then there is no efficient learning algorithm with non-trivial performance on $\eta$-almost realizable distributions.

To be self contained, and since Theorem 2.1 is not identical to [33], we outline a proof.

Proof Assume toward a contradiction that the efficient algorithm $\mathcal{L}$ is guaranteed to return a hypothesis with error $\leq \frac{1}{2}-\frac{1}{n^{c}}$ on $\eta$-almost realizable distributions. Let $M(n)$ be the maximal number of random bits used by $\mathcal{L}$ when the examples lie in $\{ \pm 1\}^{n}$. This includes both the bits

\footnotetext{
${ }^{3} \mathrm{~A}$ weaker notion, called scattering, was used in [33].
} 
describing the examples produced by the oracle and "standard" random bits. Since $\mathcal{L}$ is efficient, $M(n)<n^{c^{\prime}}$ for some $c^{\prime}>0$. Define

$$
q(n)=n^{2 c^{\prime}+2 c} .
$$

We will derive a contradiction by showing how $\mathcal{L}$ can be used to solve $\operatorname{HALF}_{q(n)}^{\mathrm{s}-\mathrm{scat}, \eta}$. To this end, consider the algorithm $\mathcal{A}$ defined below. On input $S=\left\{\left(x_{1}, y_{1}\right), \ldots,\left(x_{m}, y_{m}\right)\right\} \subset\{ \pm 1\}^{n} \times\{ \pm 1\}$,

1. Run $\mathcal{L}$ on $S$, such that the examples' oracle generates examples by choosing random examples from $S$.

2. Let $h$ be the hypothesis that $\mathcal{L}$ returns. If $\operatorname{Err}_{S}(h) \leq \frac{1}{2}-\frac{1}{n^{c}}$, output "almost-realizable". Otherwise, output "scattered".

Next, we show that $\mathcal{A}$ solves $\operatorname{HALF}_{q(n)}^{\text {s-scat, } \eta}$. Indeed, if $\operatorname{Err}_{\text {HALF }}(S) \leq \eta$, then $\mathcal{L}$ is guaranteed to return a hypothesis with $\operatorname{Err}_{S}(h) \leq \frac{1}{2}-\frac{1}{n^{c}}$, and $\mathcal{A}$ will output "almost-realizable". What if $S$ is strongly scattered? Let $\mathcal{G} \subset\{ \pm 1\}^{\{ \pm 1\}^{n}}$ be the collection of functions that $\mathcal{L}$ might return. We note that $|\mathcal{G}| \leq 2^{n^{c^{\prime}}}$, since each hypothesis in $\mathcal{G}$ can be described by $n^{c^{\prime}}$ bits. Namely, the random bits that $\mathcal{L}$ uses and the description of the examples sampled by the oracle. Now, since $\mathcal{D}$ is strongly-scattered, by Hoeffding's bound, the probability that $\operatorname{Err}_{S}(h) \leq \frac{1}{2}-\frac{1}{n^{c}}$ for a single $h:\{ \pm 1\}^{n} \rightarrow\{ \pm 1\}$ is $\leq \exp \left(-2 \frac{q(n)}{n^{2 c}}\right)$. By the union bound, the probability that $\operatorname{Err}_{S}(h) \leq \frac{1}{2}-\frac{1}{n^{c}}$ for some $h \in \mathcal{G}$ is at most $|\mathcal{G}| \exp \left(-2 \frac{q(n)}{n^{2 c}}\right) \leq 2^{n^{c^{\prime}}-\frac{q(n)}{n^{2 c}}}=2^{n^{c^{\prime}}-n^{2 c^{\prime}}}$. It follows that the probability that $\mathcal{A}$ responds "almost-realizable" is $o(1)$.

\subsection{An overview}

For the sake of simplicity, we will first explain how to prove a weaker version of Theorem 1.3. At the end of this section we will explain how to prove Theorem 1.3 in full.

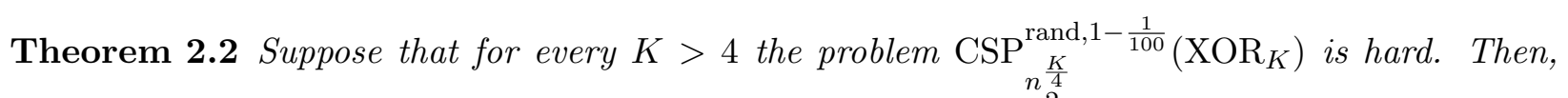
there is no efficient algorithm with non-trivial performance on $\frac{2}{100}$-almost-realizable distributions on $\{-1,1,0\}^{n} \times\{ \pm 1\}$.

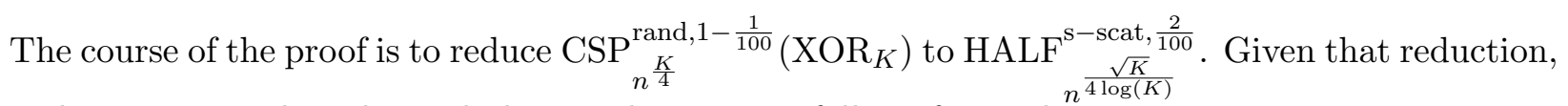
and since $K$ can be arbitrarily large, Theorem 2.2 follows from Theorem 2.1.

\section{The XOR problem as a learning problems}

The basic conceptual idea is to interpret the $K$-XOR problem as a learning problems. Every $\psi \in\{ \pm 1\}^{n}$ naturally defines $h_{\psi}: \mathcal{X}_{n, K} \rightarrow\{ \pm 1\}$, by mapping each $K$-tuple $C$ to the truth value of the corresponding constraint, given the assignment $\psi$. Namely, $h_{\psi}(C)=\mathrm{XOR}(C(\psi))$. Now, we can consider the hypothesis class $\mathcal{H}_{K}=\left\{h_{\psi} \mid \psi \in\{ \pm 1\}^{n}\right\}$.

The $K$-XOR problem can be now formulated as follows. Given $J=\left\{C_{1}, \ldots, C_{m}\right\} \subset \mathcal{X}_{n, K}$, find $h_{\psi} \in \mathcal{H}_{K}$ with minimal error on the sample $S=\left\{\left(C_{1}, 1\right), \ldots,\left(C_{m}, 1\right)\right\}$. Now, the problem $\mathrm{CSP}_{m}^{\mathrm{rand}, 1-\frac{1}{100}}\left(\mathrm{XOR}_{K}\right)$ is the problem of distinguishing between the case the $\operatorname{Err}_{\mathcal{H}_{K}}(S) \leq \frac{1}{100}$ and the case where the different $C_{i}$ 's where chosen independently and uniformly from $\mathcal{X}_{n, K}$.

The mapping $J \mapsto S$ is still not a reduction from $\operatorname{CSP}_{m}^{\text {rand,1- } \frac{1}{100}}\left(\mathrm{XOR}_{K}\right)$ to the problem of distinguishing a strongly scattered sample from a sample with small error w.r.t. halfspaces. This is due to the following points: 
- In the case that $J$ is random, $S$ is, in a sense, "very random". Yet, it is not strongly-scattered.

- We need to measure the error w.r.t. halfspaces rather than $\mathcal{H}_{K}$.

Next, we explain how we address these two points.

\section{Making the sample scattered}

Addressing the first point is relatively easy. Given a sample $\left(C_{1}, 1\right), \ldots,\left(C_{m}, 1\right)$, we can produce a new sample $\left(C_{1}^{\prime}, y_{1}^{\prime}\right), \ldots,\left(C_{m}^{\prime}, y_{m}^{\prime}\right)$ as follows: for every $i \in[m]$, w.p. $\frac{1}{2}$ we let $\left(C_{i}^{\prime}, y_{i}^{\prime}\right)=\left(C_{i}, 1\right)$ and w.p. $\frac{1}{2}$ we let $\left(C_{i}^{\prime}, y_{i}^{\prime}\right)=\left(C_{i}^{\prime},-1\right)$, where $C_{i}^{\prime}$ is obtained from $C_{i}$ by flipping the sign of the first literal. It is not hard to see that this reduction maps random instances to strongly scattered instances. Also, it is not hard to see that the error of every $h_{\psi} \in \mathcal{H}_{K}$ does not change when moving from the original sample to the new sample. Therefore, samples with error $\leq \frac{1}{100}$ are mapped to samples with error $\leq \frac{1}{100}$.

Note that the reduction not only maps random instances to scattered instances, but in fact has a stronger property that will be useful for addressing the second point (Replacing $\mathcal{H}_{K}$ by HALF). Concretely, if the original instance is random, then the $\left(C_{i}^{\prime}, y_{i}^{\prime}\right)$ 's are independent and uniform elements from $\mathcal{X}_{n, K} \times\{ \pm 1\}$.

\section{Replacing $\mathcal{H}_{K}$ by HALF}

To address the second point, we will show a reduction that maps a sample $S=\left\{\left(C_{1}, y_{1}\right), \ldots,\left(C_{m}, y_{m}\right)\right\} \subset$ $\mathcal{X}_{n, K} \times\{ \pm 1\}$ to a sample $\left(x_{1}, y_{1}\right), \ldots,\left(x_{m}, y_{m}\right) \in\{-1,1,0\}^{n^{\sqrt{K} \log (K)}} \times\{ \pm 1\}$. It will be convenient to give the reduction the option to output "not-random" instead of producing a new sample. For large enough $K$, if $m=n^{\frac{K}{4}}$, the reduction has the following properties:

- If the original sample is random (i.e., the examples $\left(C_{i}, y_{i}\right)$ where chosen independently and uniformly from $\mathcal{X}_{n, K} \times\{ \pm 1\}$ ), then w.h.p. the reduction will produce a new sample, and given that a new sample was indeed produced, it will be strongly scattered.

- If the original sample has error $\leq \frac{1}{100}$ w.r.t. $\mathcal{H}_{K}$ then the reduction will either say that the original sample is not random, or it will produce a new sample with error $\leq \frac{2}{100}$ w.r.t. halfspaces.

Putting $n^{\prime}=n^{\sqrt{K} \log (K)}$ and noting that $m=\left(n^{\prime}\right)^{\frac{\sqrt{K}}{4 \log (K)}}$, it is not hard to see that such a reduction, together with the previous reduction (the "scattering reduction"), indeed reduces $\mathrm{CSP}_{n}{ }_{n}^{\frac{K}{4}}{ }^{\mathrm{rand}, 1-\frac{1}{100}}\left(\mathrm{XOR}_{K}\right)$ to $\mathrm{HALF}^{\mathrm{s}-\mathrm{scat}, \frac{2}{100}}$.

The reduction will work as follows. It will first test that $J:=\left\{C_{1}, \ldots, C_{m}\right\}$ is pseudo random, in the sense that is satisfies certain (efficiently verifiable) properties (that will be specified later) that are possessed by random formulas w.h.p. If the test fails, the reduction will say that $S$ is not random. Otherwise, the reduction will produce the sample $\chi(S):=\left(\chi\left(C_{1}\right), y_{1}\right), \ldots,\left(\chi\left(C_{m}\right), y_{m}\right) \in$ $\{-1,1,0\}^{n^{\sqrt{K} \log (K)}} \times\{ \pm 1\}$, for a mapping $\chi: \mathcal{X}_{n, K} \rightarrow\{-1,1,0\}^{n^{\sqrt{K} \log (K)}}$ that we will specify later.

Next, we explain why this reduction have the desired properties. We start with the case that $S$ is sandom. In that case, the pseudo-randomness test will pass w.h.p. and the reduction will produce a new sample. Also, since the properties tested in this test do not depend on the labels, given that a new sample was indeed produced, the new sample is strongly scattered. 
We next deal with the case that $\operatorname{Err}_{\mathcal{H}_{K}}(S) \leq \frac{1}{100}$. We will show that in this case either the reduction will say that $S$ is not random, or the new sample will have error $\leq \frac{2}{50}$ w.r.t. halfspaces. To this end we will finally specify $\chi$ and describe the list of pseudo-random properties.

It will be convenient to define $\chi$ as a composition $\chi=\rho \circ \pi$ where $\pi: \mathcal{X}_{n, K} \rightarrow\{-1,1,0\}^{n K}$ and $\rho:\{-1,1,0\}^{n K} \rightarrow\{-1,1,0\}^{n^{\sqrt{K} \log (K)}}$. We first define $\pi$. The indicator vector of a literal is the vector in $\{0,-1,1\}^{n}$ whose all coordinates are zero except the coordinate corresponding to the literal, that is $1(-1)$ if the literal is un-negated (negated). We define $\pi(C)$ as a concatenation of $K$ vectors, where the $i$ 's vector is the indicator vector of the $i$ 'th literal in $C$. As for $\rho$, we let $\rho(x) \in\{-1,1,0\}^{n^{\sqrt{K}} \log (K)}$ be a vector consisting of all products of the form $x_{i_{1}} \cdot \ldots \cdot x_{i_{r}}$ for $r \leq \frac{1}{2} \sqrt{K} \log (K)$, and padded with zeros in the remaining coordinates. We note that for large enough $n$, the number of such products is $\leq(n K+1)^{\frac{1}{2} \sqrt{K} \log (K)} \leq n^{\sqrt{K} \log (K)}$.

Now, it is not hard to see that the error of $\chi(S)$ w.r.t. halfsapaces is exactly the error of $\pi(S)$ w.r.t. $\mathrm{POL}_{d}$ - the hypothesis class consisting of threshold functions of polynomials of degree at most $d=\frac{1}{2} \sqrt{K} \log (K)$. Therefore, we will want to show that if $\operatorname{Err}_{\mathcal{H}_{K}}(S) \leq \frac{1}{100}$ then $\operatorname{Err}_{\mathrm{POL}_{d}}(\pi(S)) \leq$ $\frac{2}{100}$.

Suppose that $\operatorname{Err}_{\mathcal{H}_{K}}(S) \leq \frac{1}{100}$, and let $\psi \in\{ \pm 1\}^{n}$ such that $\operatorname{Err}_{h_{\psi}}(S) \leq \frac{1}{100}$. To show that $\operatorname{Err}_{\mathrm{POL}_{d}}(\pi(S)) \leq \frac{2}{100}$ it is enough to construct a degree $\leq d$ polynomial $p:\{-1,1,0\}^{n K} \rightarrow \mathbb{R}$ such that $\operatorname{Pr}_{j \sim[m]}\left(h_{\psi}\left(C_{j}\right) \neq \operatorname{sign}\left(p\left(\pi\left(C_{j}\right)\right)\right) \leq \frac{1}{100}\right.$. Indeed, in that case,

$$
\begin{aligned}
\operatorname{Err}_{\mathrm{POL}_{d}}(\pi(S)) & \leq \operatorname{Pr}_{j \sim[m]}\left(y_{j} \neq \operatorname{sign}\left(p\left(\pi\left(C_{j}\right)\right)\right)\right) \\
& \leq \underset{j \sim[m]}{\operatorname{Pr}}\left(y_{j} \neq h_{\psi}\left(C_{j}\right)\right)+\underset{j \sim[m]}{\operatorname{Pr}}\left(h_{\psi}\left(C_{j}\right) \neq \operatorname{sign}\left(p\left(\pi\left(C_{j}\right)\right)\right)\right) \\
& \leq \operatorname{Err}_{h_{\psi}}(S)+\frac{1}{100} \leq \frac{1}{100}+\frac{1}{100}=\frac{2}{100}
\end{aligned}
$$

We note that we will actually find $p$ with a stronger property - namely, that $\operatorname{Pr}_{j \sim[m]}\left(h_{\psi}\left(C_{j}\right) \neq p\left(\pi\left(C_{j}\right)\right)\right) \leq$ $\frac{1}{100}$. This stronger property is not needed for proving the simplified version (Theorem 2.2), but will be needed for proving Theorem 1.3.

Unfortunately, it is not the case that we can always find such a polynomial. To overcome it, as explained above, the reduction will first check a set of pseudo-random properties. Concretely, for all small enough sets of literals, the reduction will check that the number of $K$-tuples that contain all the literals in the set is close to what is expected for a random sample. For example, it will check that the fraction of $K$-tuples containing the literal $x_{7}$ is approximately $\frac{K}{2 n}$.

It therefore remains to show that for pseudo-random $S$ there is degree $\leq d$ polynomial $p$ : $\{-1,1,0\}^{n K} \rightarrow \mathbb{R}$ such that $\operatorname{Pr}_{j \sim[m]}\left(h_{\psi}\left(C_{j}\right) \neq p\left(\pi\left(C_{j}\right)\right)\right) \leq \frac{1}{100}$. To this end, consider the linear map $T_{\psi}: \mathbb{R}^{n K} \rightarrow \mathbb{R}^{K}$ that is defined as

$$
T_{\psi}\left(v_{1}\left|v_{2}\right| \ldots \mid v_{K}\right)=\left(\left\langle v_{1}, \psi\right\rangle,\left\langle v_{2}, \psi\right\rangle, \ldots,\left\langle v_{K}, \psi\right\rangle\right)
$$

We note that $\forall C \in \mathcal{X}_{n, K}, T_{\psi}(\pi(C))=C(\psi)$. We will consider polynomials of the form $p=p^{\prime} \circ T_{\psi}$ where $p^{\prime}:\{ \pm 1\}^{K} \rightarrow \mathbb{R}$ is a degree $\leq d$ polynomial. We note that for such $p$ we have

$$
\left.\operatorname{Pr}_{j \sim[m]}\left(h_{\psi}\left(C_{j}\right) \neq p\left(\pi\left(C_{j}\right)\right)\right)=\underset{j \sim[m]}{\operatorname{Pr}}\left(\operatorname{XOR}\left(C_{j}(\psi)\right) \neq p^{\prime}\left(C_{j}(\psi)\right)\right)\right)
$$

It is therefore enough to find a degree $\leq d$ polynomial $p^{\prime}$ for which $\operatorname{Pr}_{z \sim \mathcal{D}(\psi)}\left(\operatorname{XOR}(z)=p^{\prime}(z)\right) \geq$ 0.99 . Here, $\mathcal{D}(\psi)$ is the distribution of the random variable $C_{j}(\psi)$ where $j \sim[m]$. Now, even though it is not possible to do that for general distributions, the fact that the sample is pseudo-random implies that $\mathcal{D}(\psi)$ is close, in a certain sense, to the uniform distribution on $\{ \pm 1\}^{K}$. Now, for 
uniform $z \in\{ \pm 1\}^{K}$ we have that $\left|\sum_{i=1}^{K} z_{i}\right| \leq \frac{1}{2} \sqrt{K} \log (K)$ w.p. $1-o_{K}(1)$, and the same holds for $z \sim \mathcal{D}(\psi)$. Therefore, since $\operatorname{XOR}(z)$ is fully determined given $\sum_{i=1}^{K} z_{i}$, and since $\sum_{i=1}^{K} z_{i}$ takes $\leq d$ values in the interval $[-d, d]$, we can take $p^{\prime}(z)=p^{\prime \prime}\left(\sum_{i=1}^{K} z_{i}\right)$ where $p^{\prime \prime}: \mathbb{R} \rightarrow \mathbb{R}$ is a degree $\leq \frac{1}{2} \sqrt{K} \log (K)$ polynomial that satisfies $p^{\prime \prime}\left(\sum_{i=1}^{K} z_{i}\right)=\operatorname{XOR}(z)$ whenever $\left|\sum_{i=1}^{K} z_{i}\right| \leq \sqrt{K} \log (K)$.

\section{Proving Theorem 1.3 in full}

Theorem 2.2 differs from Theorem 1.3 in two aspects. The first is that in Theorem 1.3 the completeness parameter in the assumption can be arbitrarily close to $\frac{1}{2}$ (rather than 0.99 in Theorem 2.2) and at the same time, the conclusion holds for $\eta$-almost realizable distributions for arbitrarily small $\eta$ (rather than $\eta=0.02$ in Theorem 2.2). The second aspect, that is much more minor, is that in Theorem 2.2 the hard distribution can be chosen to be supported in $\{-1,1,0\}^{n} \times\{ \pm 1\}$, while Theorem 1.3 is slightly more restrictive and requires that the distribution will be supported in $\{ \pm 1\}^{n} \times\{ \pm 1\}$.

To address the first aspect, we will first reduce the random XOR problem to the random majority-of- $q$-XORs problem, and then we will follow a similar (but slightly more involved) argument as the one described above. The reduction will work as follows. Given a XOR-formula with $m$ XOR-constraints, the reduction will produce a MXOR-formula with $\frac{m}{q}$ constraints, each of which is a majority of $q$ random XOR-constraints from the original formula. This reduction will amplify the completeness parameter toward 1.

To address the second aspect, we note that halfspaces on $\{-1,1,0\}^{n}$ can be realized by halfspaces on $\{ \pm 1\}^{2 n}$ using the map $\Psi:\{-1,1,0\}^{n} \rightarrow\{ \pm 1\}^{2 n}$ that is defined as follows:

$$
\Psi(x)=\left(\Psi\left(x_{1}\right), \ldots, \Psi\left(x_{n}\right)\right),
$$

where for $x \in\{0,-1,1\}, \Psi(x)= \begin{cases}(1,1) & x=1 \\ (-1,-1) & x=-1 . \text { It is not hard to see that for every } w \in \mathbb{R}^{n} \\ (-1,1) & x=0\end{cases}$ we have $h_{w}=h_{w^{\prime}} \circ \Psi$ where $w^{\prime}=\frac{1}{2}\left(w_{1}, w_{1}, \ldots, w_{n}, w_{n}\right)$. This observation shows that if there is no efficient algorithm with non-trivial performance on $\eta(n)$-almost-realizable distributions on $\{-1,1,0\}^{n} \times\{ \pm 1\}$, then if there is no efficient algorithm with non-trivial performance on $\eta\left(\frac{n}{2}\right)$ almost-realizable distributions on $\{ \pm 1\}^{n} \times\{ \pm 1\}$.

\section{A road map}

In section A.1 we analyze elementary properties of random formulas, and define accordingly a notion of a pseudo-random formula. We also show that if $J=\left\{C_{1}, \ldots, C_{m}\right\} \subset \mathcal{X}_{n, k}$ is a pseudo-random formula, then for $j \sim[m]$ and every assignment $\psi \in\{ \pm 1\}^{n}$, the distribution of the random variable $C_{j}(\psi) \in\{ \pm 1\}^{K}$ is close to the uniform distribution in a certain sense. In section A.2 we show that for pseudo-random formulas, for every every assignment $\psi \in\{ \pm 1\}^{n}$, the mapping $C_{j} \mapsto C_{j}(\psi)$ can be approximated by a low-degree polynomial. Finally, the full reduction is outlined in section A.3. In section A.4 we briefly explain how our argument can be extended to prove Theorems 1.4 and 1.5 .

Acknowledgements: Amit Daniely is a recipient of the Google Europe Fellowship in Learning Theory, and this research was supported in part by this Google Fellowship. The author thanks Uriel Feige, Vitaly Feldman, Nati Linial and Shai Shalev-Shwartz for valuable discussions. Many 
thanks to Sarah R. Ellen, Ryan O'donnel and David Witmer for telling me about their paper [3], that inspired this work.

\section{References}

[1] Michael Alekhnovich. More on average case vs approximation complexity. In Foundations of Computer Science, 2003. Proceedings. 44th Annual IEEE Symposium on, pages 298-307. IEEE, 2003.

[2] Mikhail Alekhnovich, Sanjeev Arora, and Iannis Tourlakis. Towards strong nonapproximability results in the lovász-schrijver hierarchy. In Proceedings of the thirty-seventh annual ACM symposium on Theory of computing, pages 294-303. ACM, 2005.

[3] Sarah Allen, Ryan O'Donnell, and David Witmer. How to refute a random csp? In FOCS, 2015.

[4] Edoardo Amaldi and Viggo Kann. On the approximability of minimizing nonzero variables or unsatisfied relations in linear systems. In Theoretical Computer Science, 1998.

[5] Edoardo Amaldi and Viggo Kann. On the approximability of minimizing nonzero variables or unsatisfied relations in linear systems. Theoretical Computer Science, 209(1):237-260, 1998.

[6] B. Applebaum, B. Barak, and D. Xiao. On basing lower-bounds for learning on worst-case assumptions. In Foundations of Computer Science, 2008. FOCS'08. IEEE 49th Annual IEEE Symposium on, pages 211-220. IEEE, 2008.

[7] Benny Applebaum, Boaz Barak, and Avi Wigderson. Public key cryptography from different assumptions. In STOC, 2010.

[8] Sanjeev Arora, László Babai, Jacques Stern, and Z Sweedyk. The hardness of approximate optima in lattices, codes, and systems of linear equations. In Foundations of Computer Science, 1993. Proceedings., 34th Annual Symposium on, pages 724-733. IEEE, 1993.

[9] James Aspnes, Richard Beigel, Merrick Furst, and Steven Rudich. The expressive power of voting polynomials. Combinatorica, 14(2):135-148, 1994.

[10] Pranjal Awasthi, Maria-Florina Balcan, and Phil Long. The power of localization for efficiently learning linear separators with noise. In STOC, 2014.

[11] Boaz Barak and Ankur Moitra. Tensor prediction, rademacher complexity and random 3-xor. In Arxiv preprint arXiv:1501.06521 v2, 2015.

[12] Boaz Barak, Guy Kindler, and David Steurer. On the optimality of semidefinite relaxations for average-case and generalized constraint satisfaction. In Robert D. Kleinberg, editor, ITCS, pages 197-214, 2013.

[13] Paul Beame and Toniann Pitassi. Simplified and improved resolution lower bounds. In Foundations of Computer Science, 1996. Proceedings., 37th Annual Symposium on, pages 274-282. IEEE, 1996.

[14] Paul Beame, Richard Karp, Toniann Pitassi, and Michael Saks. On the complexity of unsatisfiability proofs for random k-cnf formulas. In Proceedings of the thirtieth annual ACM symposium on Theory of computing, pages 561-571. ACM, 1998. 
[15] Richard Beigel, Nick Reingold, and Daniel Spielman. The perceptron strikes back. In Structure in Complexity Theory Conference, 1991., Proceedings of the Sixth Annual, pages 286-291. IEEE.

[16] S. Ben-David, D. Loker, N. Srebro, and K. Sridharan. Minimizing the misclassification error rate using a surrogate convex loss. In ICML, 2012.

[17] Shai Ben-David, Nadav Eiron, and Philip Long. On the difficulty of approximately maximizing agreements. In JCSS, 2003.

[18] Eli Ben-Sasson. Expansion in proof complexity. In Hebrew University. Citeseer, 2001.

[19] Eli Ben-Sasson and Avi Wigderson. Short proofs are narrowresolution made simple. In Proceedings of the thirty-first annual ACM symposium on Theory of computing, pages 517-526. ACM, 1999.

[20] E. Blais, R. O’Donnell, and K Wimmer. Polynomial regression under arbitrary product distributions. In COLT, 2008.

[21] Avrim Blum, Merrick Furst, Jeffrey Jackson, Michael Kearns, Yishay Mansour, and Steven Rudich. Weakly learning dnf and characterizing statistical query learning using fourier analysis. In Proceedings of the twenty-sixth annual ACM symposium on Theory of computing, pages 253262. ACM, 1994.

[22] Avrim Blum, Adam Kalai, and Hal Wasserman. Noise-tolerant learning, the parity problem, and the statistical query model. Journal of the ACM (JACM), 50(4):506-519, 2003.

[23] Nader Bshouty and Lynn Burroughs. Maximizing agreements and coagnostic learning. In Theoretical Computer Science, 2006.

[24] Joshua Buresh-Oppenheim, Nicola Galesi, Shlomo Hoory, Avner Magen, and Toniann Pitassi. Rank bounds and integrality gaps for cutting planes procedures. In Foundations of Computer Science, 2003. Proceedings. 44th Annual IEEE Symposium on, pages 318-327. IEEE, 2003.

[25] Amin Coja-Oghlan, Andreas Goerdt, and André Lanka. Strong refutation heuristics for random k-sat. In Approximation, Randomization, and Combinatorial Optimization. Algorithms and Techniques, pages 310-321. Springer, 2004.

[26] Amin Coja-Oghlan, Colin Cooper, and Alan Frieze. An efficient sparse regularity concept. SIAM Journal on Discrete Mathematics, 23(4):2000-2034, 2010.

[27] C. Cortes and V. Vapnik. Support-vector networks. Machine Learning, 20(3):273-297, September 1995 .

[28] N. Cristianini and J. Shawe-Taylor. An Introduction to Support Vector Machines. Cambridge University Press, 2000.

[29] N. Cristianini and J. Shawe-Taylor. Kernel Methods for Pattern Analysis. Cambridge University Press, 2004.

[30] Amit Daniely. A ptas for agnostically learning halfspaces. In COLT, 2015.

[31] Amit Daniely and Shai Shalev-Shwartz. Complexity theoretic limitations on learning dnf's. In Arxiv preprint arXiv:1404.3378 v1, 2014. 
[32] Amit Daniely, Nati Linial, and Shai Shalev-Shwartz. More data speeds up training time in learning halfspaces over sparse vectors. In NIPS, 2013.

[33] Amit Daniely, Nati Linial, and Shai Shalev-Shwartz. From average case complexity to improper learning complexity. STOC, 2014.

[34] Amit Daniely, Nati Linial, and Shai Shalev-Shwartz. The complexity of learning halfspaces using generalized linear methods. COLT, 2014.

[35] Martin Davis, George Logemann, and Donald Loveland. A machine program for theoremproving. Communications of the ACM, 5(7):394-397, 1962.

[36] Uriel Feige. Relations between average case complexity and approximation complexity. In Proceedings of the thiry-fourth annual ACM symposium on Theory of computing, pages 534543. ACM, 2002.

[37] Uriel Feige and Eran Ofek. Easily refutable subformulas of large random 3cnf formulas. In Automata, languages and programming, pages 519-530. Springer, 2004.

[38] V. Feldman, P. Gopalan, S. Khot, and A.K. Ponnuswami. New results for learning noisy parities and halfspaces. In In Proceedings of the 47th Annual IEEE Symposium on Foundations of Computer Science, 2006.

[39] Vitaly Feldman, Will Perkins, and Santosh Vempala. On the complexity of random satisfiability problems with planted solutions. In STOC, 2015.

[40] Y. Freund and R.E. Schapire. A decision-theoretic generalization of on-line learning and an application to boosting. In EUROCOLT, pages 23-37. Springer-Verlag, 1995.

[41] V. Guruswami and P. Raghavendra. Hardness of learning halfspaces with noise. In Proceedings of the 47th Foundations of Computer Science (FOCS), 2006.

[42] Armin Haken. The intractability of resolution. Theoretical Computer Science, 39:297-308, 1985.

[43] Johan Håstad. Some optimal inapproximability results. Journal of the ACM (JACM), 48(4): 798-859, 2001.

[44] A. Kalai, A.R. Klivans, Y. Mansour, and R. Servedio. Agnostically learning halfspaces. In Proceedings of the 46th Foundations of Computer Science (FOCS), 2005.

[45] Adam Tauman Kalai, Adam R Klivans, Yishay Mansour, and Rocco A Servedio. Agnostically learning halfspaces. SIAM Journal on Computing, 37(6):1777-1805, 2008.

[46] R. M. Karp. Reducibility among combinatorial problems. In R. E. Miller and J. W. Thatcher, editors, Complexity of Computer Computations, pages 85-103. Plenum Press, 1972.

[47] Michael Kearns. Efficient noise-tolerant learning from statistical queries. In STOC, pages 392-401, 1993.

[48] Michael Kearns and Ming Li. Learning in the presence of malicious errors. SIAM Journal on Computing, 22(4):807-837, August 1993. 
[49] Michael Kearns and Leslie G. Valiant. Cryptographic limitations on learning Boolean formulae and finite automata. In STOC, pages 433-444, May 1989.

[50] Adam Klivan and Pravesh Kothari. Embedding hard learning problems in gaussian space. In RANDOM, 2014.

[51] Adam R. Klivans and Alexander A. Sherstov. Cryptographic hardness for learning intersections of halfspaces. In FOCS, 2006.

[52] N. Linial and Z. Luria. Chernoff's Inequality - A very elementary proof. Arxiv preprint arXiv:1403.7739 v2, 2014.

[53] P.M. Long and R.A. Servedio. Learning large-margin halfspaces with more malicious noise. In NIPS, 2011.

[54] L. Pitt and L.G. Valiant. Computational limitations on learning from examples. Journal of the Association for Computing Machinery, 35(4):965-984, October 1988.

[55] F. Rosenblatt. The perceptron: A probabilistic model for information storage and organization in the brain. Psychological Review, 65:386-407, 1958. (Reprinted in Neurocomputing (MIT Press, 1988).).

[56] F. Rosenblatt. Principles of Neurodynamics. Spartan, New York, 1962.

[57] R.E. Schapire. The strength of weak learnability. In FOCS, pages 28-33, October 1989.

[58] Robert E Schapire and Yoav Freund. Boosting: Foundations and algorithms. MIT press, 2012.

[59] Grant Schoenebeck. Linear level lasserre lower bounds for certain k-csps. In Foundations of Computer Science, 2008. FOCS'08. IEEE 49th Annual IEEE Symposium on, pages 593-602. IEEE, 2008.

[60] B. Schölkopf and A. J. Smola. Learning with Kernels: Support Vector Machines, Regularization, Optimization and Beyond. MIT Press, 2002.

[61] V. N. Vapnik. Statistical Learning Theory. Wiley, 1998.

\section{A Proof of Theorem 1.3}

\section{A.1 Pseudo-random formulas}

A partial $K$-tuple supported in $A \subset[K]$ is a mapping $C:\{ \pm 1\}^{n} \rightarrow\{-1,1, *\}^{K}$ such that the output coordinates corresponding to $A$ are literals corresponding to $|A|$ different variables, and the remaining coordinates are the constant function $*$. The size of $C$ is $|A|$. We denote by $\mathcal{X}_{n, K, A}$ the collection of partial $K$-tuples that are supported in $A$. We note that

$$
\left|\mathcal{X}_{n, K, A}\right|=(2 n)(2 n-2) \cdot \ldots \cdot(2 n+2-2|A|)
$$

For $A \subset[K]$ we denote by $\Pi_{A}:\{ \pm 1\}^{K} \rightarrow\{-1,1, *\}^{K}$ the function that maps all coordinates in $[K] \backslash A$ to $*$ and leaves the remaining coordinates unchanged. For $C \in \mathcal{X}_{n, K}$ we denote by $C_{A}:\{ \pm 1\}^{n} \rightarrow\{-1,1, *\}^{K}$ the partial $K$-tuple $\Pi_{A} \circ C$. For a $K$-formula $J$, and a partial $K$-tuple $C$ we define the frequency of $C$ in $J$ as $\operatorname{Fr}_{J}(C)=\frac{\left|\left\{C^{\prime} \in J: C_{A}^{\prime}=C\right\}\right|}{m}$. We note that the for random $J$, if $C$ is 
a partial $K$-tuple that is supported in a set $A$ of size $t$, then $\operatorname{Fr}_{J}(C)$ is an average of $m$ independent Bernulli variables with parameter $p_{n, t}:=\frac{1}{\left|\mathcal{X}_{n, K, A}\right|}=\frac{1}{(2 n)(2 n-2) \cdot \ldots \cdot(2(n-t+1))}$. By Hoeffding's bound we have

Lemma A.1 Let $J=\left\{C_{1}, \ldots, C_{m}\right\}$ be a random formula. Then, for every partial $K$-tuple of size $t$ we have $\operatorname{Pr}_{J}\left(\left|\operatorname{Fr}_{J}(C)-p_{n, t}\right| \geq \tau\right) \leq 2 \exp \left(-2 m \tau^{2}\right)$

We say that $J$ is $(t, \tau)$-pseudo-random if $\left|\operatorname{Fr}_{J}(C)-p_{n, t^{\prime}}\right|<\tau$ for every partial $K$-tuple $C$ of size $t^{\prime} \leq t$. We say that $J$ is $\tau$-pseudo-random if it is $(K, \tau)$-pseudo-random. By lemma A.1, the fact that the number of partial $K$-tuples is

$$
1+\sum_{j=1}^{K}\left(\begin{array}{c}
K \\
j
\end{array}\right)(2 n)(2 n-2) \cdot \ldots \cdot(2 n+2-2 j) \leq 2^{K}(2 n)^{K} \leq(2 n)^{2 K},
$$

and the union bound we have

Lemma A.2 Let $J=\left\{C_{1}, \ldots, C_{m}\right\}$ be a random formula. Then the probability that $J$ is not $\tau$-pseudo-random is at most $(2 n)^{2 K} 2 \exp \left(-2 m \tau^{2}\right)$

For a formula $J=\left\{C_{1}, \ldots, C_{m}\right\} \subset \mathcal{X}_{n, K}$ and $\psi \in\{ \pm 1\}^{n}$, we denote by $\mathcal{D}(J, \psi)$ the distribution of the random variable $C_{j}(\psi) \in\{ \pm 1\}^{K}$ where $j \sim \operatorname{Uni}([m])$. A vector $z \in\{-1,1, *\}^{K}$ is supported in $A \subset[K]$ if $A=\left\{i \in[K] \mid z_{i} \neq *\right\}$. We say that a distribution $\mathcal{D}$ on $\{ \pm 1\}^{K}$ is $(t, \mu)$-close to the uniform distribution if for every $z \in\{-1,1, *\}^{K}$ that is supported in a set $A \in\left(\begin{array}{c}{[k]} \\ \leq t\end{array}\right)$ we have that $\left|\operatorname{Pr}_{z^{\prime} \sim \mathcal{D}}\left(\Pi_{A}\left(z^{\prime}\right)=z\right)-2^{-|A|}\right| \leq \mu$

Lemma A.3 If $J$ is $(t, \tau)$-pseudo-random then for every $\psi \in\{ \pm 1\}^{n}, \mathcal{D}(J, \psi)$ is $\left(t, n^{t} \tau\right)$-close to the uniform distribution.

Proof Fix a vector $z \in\{-1,1, *\}^{K}$ supported in $A \in\left(\begin{array}{c}{[K]} \\ \leq t\end{array}\right)$. We have

$$
\begin{aligned}
\underset{z^{\prime} \sim \mathcal{D}(J, \psi)}{\operatorname{Pr}}\left(\Pi_{A}\left(z^{\prime}\right)=z\right) & =\operatorname{Pr}_{j \sim \operatorname{Uni}([m])}\left(\left(C_{j}\right)_{A}(\psi)=z\right) \\
& =\frac{1}{m}\left|\left\{j \in[m] \mid\left(C_{j}\right)_{A}(\psi)=z\right\}\right| \\
& =\frac{1}{m} \sum_{C \in \mathcal{X}_{n, K, A} \mid C(\psi)=z}\left|\left\{j \in[m] \mid\left(C_{j}\right)_{A}=C\right\}\right| \\
& =\sum_{C \in \mathcal{X}_{n, K, A} \mid C(\psi)=z} \operatorname{Fr}_{J}(C)
\end{aligned}
$$

Denote $U=\left\{C \in \mathcal{X}_{n, K, A} \mid C(\psi)=z\right\}$ and note that $|U|=n(n-1) \cdot \ldots \cdot(n-|A|+1)=\frac{\left|\mathcal{X}_{n, K, A}\right|}{2^{|A|}}$. By the $(t, \tau)$-pseudo-randomness of $J$ we have

$$
\begin{aligned}
\operatorname{Pr}_{j \sim \operatorname{Uni}([m])}\left(\left(C_{j}\right)_{A}(\psi)=z\right)-2^{-|A|} \mid & =\left|\sum_{C \in U} \operatorname{Fr}_{J}(C)-2^{-|A|}\right| \\
& \leq\left|\sum_{C \in U} p_{n,|A|}-2^{-|A|}\right|+|U| \tau \\
& =|| U\left|p_{n,|A|}-2^{-|A|}\right|+|U| \tau=|U| \tau \leq n^{|A|} \tau \leq n^{t} \tau
\end{aligned}
$$




\section{A.2 Approximately realizing assignments by polynomials}

In this section we will show that when a formula $J=\left\{C_{1}, \ldots, C_{m}\right\}$ is pseudo-random then for every assignment $\psi \in\{ \pm 1\}^{n}$ the mapping $C \mapsto \mathrm{XOR}(C(\psi))$ can be approximately realized by a low-degree polynomial on $J$. Namely, there is a low degree polynomial $p: \mathcal{X}_{n, K} \rightarrow\{ \pm 1\}$ such that on most $K$-tuples $C \in J$, we have $p(C)=\operatorname{XOR}(C(\psi))$. To this end, we must represent $K$-tuples as vectors. The way we will do this is the following. Recall that the indicator vector of a literal is the vector in $\{0,-1,1\}^{n}$ whose all coordinates are zero except the coordinate corresponding to the literal, that is $1(-1)$ if the literal is un-negated (negated). Also, we defined $\pi: \mathcal{X}_{n, K} \rightarrow\{0,-1,1\}^{n K}$ such that $\pi(C)$ is a concatenation of $K$ vectors, where the $i$ 's vector is the indicator vector of the $i$ 'th literal in $C$.

We will use the following version of Chernoff's bound from [52].

Lemma A.4 Let $\mathcal{D}$ be a distribution on $\{ \pm 1\}^{K}, 1 \geq \beta>0$ and $\frac{1}{2} \leq \alpha<\frac{1+\beta}{2}$. Assume that for every $z \in\{-1,1, *\}$ that is supported in a set $A$ of size $r=\lceil\beta K\rceil$ we have $\operatorname{Pr}_{z^{\prime} \sim \mathcal{D}}\left(\Pi_{A}\left(z^{\prime}\right)=z\right) \leq \alpha^{r}$. Then

$$
\operatorname{Pr}_{z \sim \mathcal{D}}\left(\left|\sum_{i=1}^{K} z_{i}\right| \geq \beta K\right) \leq 2 \exp \left(-D\left(\frac{1+\beta}{2}, \alpha\right) K\right)
$$

Lemma A.5 Let $\mathcal{D}$ be a distribution on $\{ \pm 1\}^{K}$ that is $(t, \mu)$-close to the uniform distribution, and let $d$ such that $\frac{K 2^{d} \mu}{d}<d \leq t$. Then, there exists a degree $\leq d$ polynomial $p:\{ \pm 1\}^{K} \rightarrow \mathbb{R}$ such that

$$
\operatorname{Pr}_{z \sim \mathcal{D}}(\operatorname{XOR}(z) \neq p(z)) \leq 2 \exp \left(-D\left(\frac{1}{2}+\frac{d}{2 K}, \frac{1}{2}+\frac{2^{d-1} \mu}{d}\right) K\right)
$$

Proof Let $\Lambda:\{ \pm 1\}^{K} \rightarrow \mathbb{R}$ be the linear mapping $\Lambda(z)=\sum_{i=1}^{K} z_{i}$. We note that XOR $(z)$ is fully determined given $\Lambda(z)$. Namely there is $f: \mathbb{R} \rightarrow\{ \pm 1\}$ such that XOR $=f \circ \Lambda$. We note that the image of $\Lambda$ has at most $d+1$ values in the interval $[-d, d]$. Therefore, there is a degree $\leq d$ polynomial $q: \mathbb{R} \rightarrow \mathbb{R}$ that coincides with $f$ on $\Lambda\left(\{ \pm 1\}^{K}\right) \cap[-d, d]$. Consider now the degree $\leq d$ polynomial $p=q \circ \Lambda$. We have that $p$ coincides with XOR whenever $\left|\sum_{i=1}^{K} z_{i}\right| \leq d$. Hence, we have

$$
\operatorname{Pr}_{z \sim \mathcal{D}}(\operatorname{XOR}(z) \neq p(z)) \leq \operatorname{Pr}_{z \sim \mathcal{D}}\left(\left|\sum_{i=1}^{K} z_{i}\right| \geq d\right) .
$$

The lemma will now follow from Lemma A.4 when $\beta=\frac{d}{K}$ and $\alpha=\frac{1}{2}+\frac{2^{d-1} \mu}{d}$. It remains to show that the conditions of the Lemmas hold, namely, that $\alpha<\frac{1+\beta}{2}$ and that for every $z \in\{-1,1, *\}$ that is supported in a set $A$ of size $r=\lceil\beta K\rceil=d$ we have $\operatorname{Pr}_{z^{\prime} \sim \mathcal{D}}\left(\Pi_{A}\left(z^{\prime}\right)=z\right) \leq \alpha^{d}$. The first conditions follows from the requirement that $\frac{K 2^{d} \mu}{d}<d$. For the second condition, since $\mathcal{D}$ is $(t, \mu)$-close to the uniform distribution and $d \leq t$ we have

$$
\begin{aligned}
\alpha^{d} & =\sum_{i=0}^{d}\left(\begin{array}{l}
d \\
i
\end{array}\right)\left(\frac{1}{2}\right)^{d-i}\left(\frac{2^{d-1} \mu}{d}\right)^{i} \\
& \geq\left(\frac{1}{2}\right)^{d}+d\left(\frac{1}{2}\right)^{d-1}\left(\frac{2^{d-1} \mu}{d}\right) \\
& =\left(\frac{1}{2}\right)^{d}+\mu \\
& \geq \operatorname{Pr}_{z^{\prime} \sim \mathcal{D}}\left(\Pi_{A}\left(z^{\prime}\right)=z\right)
\end{aligned}
$$


Lemma A.6 Let $J=\left\{C_{1}, \ldots, C_{m}\right\} \in \mathcal{X}_{n, K}$ be $(t, \tau)$-pseudo-random, $\psi \in\{ \pm 1\}^{n}$ and $d$ such that $\frac{K 2^{d} n^{d} \tau}{d}<d \leq t$. Then, there exists a degree $\leq d$ polynomial $p:\{0,-1,1\}^{n K} \rightarrow \mathbb{R}$ such that

$$
\underset{j \sim[m]}{\operatorname{Pr}}\left(\operatorname{XOR}\left(C_{j}(\psi)\right) \neq p\left(\pi\left(C_{j}\right)\right)\right) \leq 2 \exp \left(-D\left(\frac{1}{2}+\frac{d}{2 K}, \frac{1}{2}+\frac{2^{d-1} n^{d} \tau}{d}\right) K\right)
$$

Proof Since $J$ is $(t, \tau)$-pseudo-random and $d \leq t$, it is also $(d, \tau)$-pseudo-random. By Lemma A.3 $\mathcal{D}(J, \psi)$ is $\left(d, n^{d} \tau\right)$-close to the uniform distribution. Therefore, by Lemma A.5 there is degree $\leq d$ polynomial $p^{\prime}$ for which

$$
\underset{z \sim \mathcal{D}(J, \psi)}{\operatorname{Pr}}\left(\operatorname{XOR}(z) \neq p^{\prime}(z)\right) \leq 2 \exp \left(-D\left(\frac{1}{2}+\frac{d}{2 K}, \frac{1}{2}+\frac{2^{d-1} n^{d} \tau}{d}\right) K\right) .
$$

Now, let $T: \mathbb{R}^{n K} \rightarrow \mathbb{R}^{K}$ be the linear map

$$
T\left(v_{1}\left|v_{2}\right| \ldots \mid v_{K}\right)=\left(\left\langle v_{1}, \psi\right\rangle,\left\langle v_{2}, \psi\right\rangle, \ldots,\left\langle v_{K}, \psi\right\rangle\right)
$$

Note that for all $C \in \mathcal{X}_{n, K}, T(\pi(C))=C(\psi)$. Consider the degree $\leq d$ polynomial $p=p^{\prime} \circ T$. We have

$$
\begin{aligned}
\operatorname{Pr}_{j \sim[m]}\left(\operatorname{XOR}\left(C_{j}(\psi)\right) \neq p\left(\pi\left(C_{j}\right)\right)\right) & =\operatorname{Pr}_{j \sim[m]}\left(\operatorname{XOR}\left(C_{j}(\psi)\right) \neq p^{\prime}\left(T\left(\pi\left(C_{j}\right)\right)\right)\right) \\
& =\operatorname{Pr}_{j \sim[m]}\left(\operatorname{XOR}\left(C_{j}(\psi)\right) \neq p^{\prime}\left(C_{j}(\psi)\right)\right) \\
& =\operatorname{Pr}_{z \sim \mathcal{D}(J, \psi)}\left(\operatorname{XOR}(z) \neq p^{\prime}(z)\right) \\
& \leq 2 \exp \left(-D\left(\frac{1}{2}+\frac{d}{2 K}, \frac{1}{2}+\frac{2^{d-1} n^{d} \tau}{d}\right) K\right)
\end{aligned}
$$

\section{A.3 The reduction}

\section{Step I: Amplifying the gap - from XOR to majority of XORs}

For odd $q$, we define the predicate $\operatorname{MXOR}_{q, K}:\{ \pm 1\}^{q K} \rightarrow\{ \pm 1\}$ by

$$
\operatorname{MXOR}_{q, K}(z)=\operatorname{MAJ}\left(\operatorname{XOR}\left(z_{1}, \ldots, z_{k}\right), \ldots, \operatorname{XOR}\left(z_{(q-1) K+1}, \ldots, z_{q K}\right)\right)
$$

A $(q, K)$-tuple is an element in $\mathcal{X}_{n, q, K}:=\left(\mathcal{X}_{n, K}\right)^{q}$. For a $(q, K)$-tuple $C=\left(C^{1}, \ldots, C^{q}\right)$ and an assignment $\psi \in\{ \pm 1\}^{n}$ we denote $C(\psi)=\left(C^{1}(\psi), \ldots, C^{q}(\psi)\right) \in\{ \pm 1\}^{q K}$. A $(q, K)$-formula is a collection $J=\left\{C_{1}, \ldots, C_{m}\right\}$ of $(q, K)$-tuples. An instance to the $(q, K)$-MXOR problem is a $(q, K)$-formula, and the goal is to find an assignment $\psi \in\{ \pm 1\}^{n}$ that maximizes $\operatorname{VAL}_{\psi, \mathrm{MXOR}}(J):=$ $\frac{\left|\left\{j: \operatorname{MXOR}_{q, K}\left(C_{i}(\psi)\right)=1\right\}\right|}{m}$. We define the value of $J$ as $\operatorname{VAL}_{\text {MXOR }}(J):=\max _{\psi \in\{ \pm 1\}^{n}} \operatorname{VAL}_{\psi, \operatorname{MXOR}}(J)$.

For $m=m(n),(q, K)=(q(n), K(n))$ and $\frac{1}{2}>\eta=\eta(n)>0$, we say that the problem $\mathrm{CSP}_{m}^{\mathrm{rand}, 1-\eta}\left(\mathrm{MXOR}_{q, K}\right)$ is easy, if there exists an efficient randomized algorithm, $\mathcal{A}$ with the following properties. Its input is a $(q, K)$-formula $J$ with $n$ variables and $m$ constraints and its output satisfies:

- If $\operatorname{VAL}_{\mathrm{MXOR}}(J) \geq 1-\eta$, then

$$
\operatorname{Pr}_{\text {coins of } \mathcal{A}}(\mathcal{A}(J)=\text { "non-random" }) \geq \frac{3}{4}
$$


- If $J$ is random ${ }^{4}$ then, with probability $1-o_{n}(1)$ over the choice of $J$,

$$
\operatorname{Pr}_{\text {coins of } \mathcal{A}}\left(\mathcal{A}(J)=\text { "random") } \geq \frac{3}{4} .\right.
$$

Lemma A.7 The problem $\mathrm{CSP}_{m}^{\mathrm{rand}, \eta}\left(\mathrm{XOR}_{K}\right)$ can be efficiently reduced to $\mathrm{CSP}_{\left\lfloor\frac{m}{q}\right\rfloor}^{\mathrm{rand}, 1-4 \exp \left(-2\left(\eta-\frac{1}{2}\right)^{2} q\right)}\left(\mathrm{MXOR}_{q, K}\right)$. We will use the following version of Chernoff's bound from [52].

Lemma A.8 Let $\mathcal{D}$ be a distribution on $\{0,1\}^{q}$ and $\eta<\frac{1}{2}$. Assume that for every $A \subset[q]$ we have $\operatorname{Pr}_{z \sim \mathcal{D}}\left(\forall i \in A, z_{i}=1\right) \leq \eta^{|A|}$. Then

$$
\operatorname{Pr}_{z \sim \mathcal{D}}\left(\sum_{i=1}^{q} z_{i} \geq \frac{q}{2}\right) \leq \exp \left(-D\left(\frac{1}{2}, \eta\right) q\right) \leq \exp \left(-2\left(\frac{1}{2}-\eta\right)^{2} q\right)
$$

Proof (of Lemma A.7) Given a $K$-formula $J=\left\{C_{1}, \ldots, C_{m}\right\} \in \mathcal{X}_{n, K}$ we will produce a $(q, K)$ formula $J^{\prime}$ as follows. We first randomly throw $m-m\left\lfloor\frac{m}{q}\right\rfloor$ of $J$ 's $K$-tuples. Then, we randomly partition the remaining tuples into $\left\lfloor\frac{m}{q}\right\rfloor$ equally sized ordered bundles. For each such bundle $\left\{C_{j_{1}}, \ldots, C_{j_{q}}\right\}$, we add to $J^{\prime}$ the $(q, K)$-tuple $C=\left(C_{j_{1}}, \ldots, C_{j_{q}}\right)$. To see that the reduction works note that:

- If $J$ is random then so is $J^{\prime}$.

- Assume now that $\operatorname{VAL}_{\mathrm{XOR}}(J) \geq 1-\eta$, and let $\psi \in\{ \pm 1\}^{n}$ be an assignment with $\operatorname{VAL}_{\psi, \mathrm{XOR}}(J) \geq$ $1-\eta$. Consider a single random bundle $\left\{C_{j_{1}}, \ldots, C_{j_{q}}\right\}$. By Lemma A.8 we have that the probability that for most $K$-tuples in the bundle we have $\operatorname{XOR}_{K}(C(\psi))=-1$ is $\leq$ $\exp \left(-2\left(\eta-\frac{1}{2}\right)^{2} q\right)$. Hence, $E\left[1-\operatorname{VAL}_{\mathrm{MXOR}}\left(J^{\prime}\right)\right] \leq \exp \left(-2\left(\eta-\frac{1}{2}\right)^{2} q\right)$. By Markov's inequality we have that $\operatorname{VAL}_{\mathrm{MXOR}}\left(J^{\prime}\right) \geq 1-4 \exp \left(-2\left(\eta-\frac{1}{2}\right)^{2} q\right)$ w.p. $\geq \frac{3}{4}$.

\section{Step II: Making the sample scattered - from (MXOR) to (MXOR, $\neg$ MXOR)}

A labeled $(q, K)$-formula is a collection $J=\left\{\left(C_{1}, y_{1}\right) \ldots,\left(C_{m}, y_{m}\right)\right\} \subset \mathcal{X}_{n, q, K}$. An instance to the $(q, K)$-(MXOR, $\neg$ MXOR) problem is a labeled $(q, K)$-formula, and the goal is to find an assignment $\psi \in\{ \pm 1\}^{n}$ that maximizes $\operatorname{VAL}_{\psi, \operatorname{MXOR}}(J):=\frac{\left|\left\{j: \operatorname{MXOR}_{q, K}\left(C_{i}(\psi)\right)=y_{i}\right\}\right|}{m}$. We define the value of $J$ as $\operatorname{VAL}_{\text {MXOR }}(J):=\max _{\psi \in\{ \pm 1\}^{n}} \operatorname{VAL}_{\psi, \operatorname{MXOR}}(J)$. For $m=m(n),(q, K)=(q(n), K(n))$ and $\frac{1}{2}>\eta=\eta(n)>0$, we say that the problem $\operatorname{CSP}_{m}^{\mathrm{rand}, 1-\eta}\left(\mathrm{MXOR}_{q, K}, \neg \mathrm{MXOR}_{q, K}\right)$ is easy, if there exists an efficient randomized algorithm, $\mathcal{A}$ with the following properties. Its input is a labeled $(q, K)$-formula $J$ with $n$ variables and $m$ constraints and its output satisfies:

- If $\operatorname{VAL}_{\mathrm{MXOR}}(J) \geq 1-\eta$, then

$$
\operatorname{Pr}_{\text {coins of } \mathcal{A}}(\mathcal{A}(J)=\text { "non-random" }) \geq \frac{3}{4}
$$

\footnotetext{
${ }^{4}$ To be precise, the $(q, K)$-tuples are chosen uniformly, and independently from one another.
} 
- If $J$ is random ${ }^{5}$ then, with probability $1-o_{n}(1)$ over the choice of $J$,

$$
\operatorname{Pr}_{\text {coins of } \mathcal{A}}\left(\mathcal{A}(J)=\text { "random") } \geq \frac{3}{4} .\right.
$$

Lemma A.9 The problem $\mathrm{CSP}_{m}^{\text {rand }, 1-\eta}\left(\mathrm{MXOR}_{q, K}\right)$ can be efficiently reduced to $\mathrm{CSP}_{m}^{\text {rand }, 1-\eta}\left(\mathrm{MXOR}_{q, K}, \neg \mathrm{MXOR}_{q}\right.$,

Proof Given an instance $J=\left\{C_{1}, \ldots, C_{m}\right\}$ to $\mathrm{CSP}_{m}^{\text {rand, } 1-\eta}\left(\mathrm{MXOR}_{q, K}\right)$, we will produce an instance $J^{\prime}$ to $\mathrm{CSP}_{m}^{\text {rand }, 1-\eta}\left(\mathrm{MXOR}_{q, K}, \neg \mathrm{MXOR}_{q, K}\right)$ as follows. For each $C_{j}$, w.p. $\frac{1}{2}$ we will add to $J^{\prime}$ the pair $\left(C_{j}, 1\right)$, and w.p. $\frac{1}{2}$ we will add the pair $\left(C_{j}^{\prime},-1\right)$ where $C_{j}^{\prime}=\left(C_{j}^{\prime 1}, \ldots, C_{j}^{\prime q}\right)$ is obtained from $C_{j}=\left(C_{j}^{1}, \ldots, C_{j}^{q}\right)$ by flipping, for each $C_{j}^{i}$, the sign of the first literal. It is not hard to see that if $J$ is random then so is $J^{\prime}$. Also, for every $\psi \in\{ \pm 1\}^{n}, \operatorname{VAL}_{\psi, \operatorname{MXOR}}(J)=\operatorname{VAL}_{\psi, \operatorname{MXOR}}\left(J^{\prime}\right)$, and therefore, if $\operatorname{VAL}_{\mathrm{MXOR}}(J) \geq 1-\eta$ then $\operatorname{VAL}_{\mathrm{MXOR}}\left(J^{\prime}\right) \geq 1-\eta$ as well.

\section{Step III: Enforcing pseudo-randomness}

We say that a labeled $(q, K)$-formula $J$ is $(t, \tau)$-pseudo-random if the $K$-formula consisting of all the $K$-tuples that appear in $J$ is $(t, \tau)$-pseudo-random. For $m=m(n),(q, K)=(q(n), K(n)),(t, \tau)=$ $(t(n), \tau(n))$ and $\frac{1}{2}>\eta=\eta(n)>0$, we say that the problem $\mathrm{CSP}_{m,(t, \tau)}^{\mathrm{rand}, 1-\eta}\left(\mathrm{MXOR}_{q, K}, \neg \mathrm{MXOR}_{q, K}\right)$ is easy, if there exists an efficient randomized algorithm, $\mathcal{A}$ with the following properties. Its input is a labeled $(q, K)$-formula $J$ with $n$ variables and $m$ constraints that is $(t, \tau)$-pseudo-random. Its output satisfies:

- If $\operatorname{VAL}_{\mathrm{MXOR}}(J) \geq 1-\eta$, then

$$
\operatorname{Pr}_{\text {coins of } \mathcal{A}}(\mathcal{A}(J)=\text { "non-random" }) \geq \frac{3}{4}
$$

- If $J$ is random ${ }^{6}$ then, with probability $1-o_{n}(1)$ over the choice of $J$,

$$
\operatorname{Pr}_{\text {coins of } \mathcal{A}}\left(\mathcal{A}(J)=\text { "random") } \geq \frac{3}{4} .\right.
$$

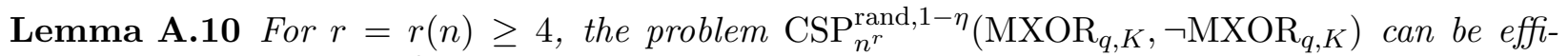
ciently reduced to $\mathrm{CSP}_{n^{r},\left(r, n^{-\frac{r}{4}}\right)}^{\mathrm{rand}, 1-\eta}\left(\mathrm{MXOR}_{q, K}, \neg \mathrm{MXOR}_{q, K}\right)$.

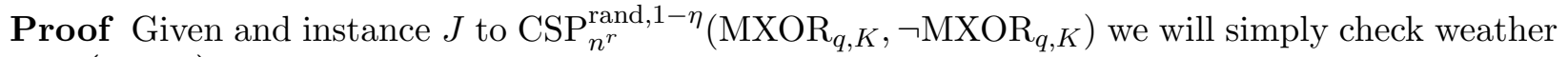
it is $\left(r, n^{-\frac{r}{4}}\right)$-pseudo-random or not. If it not, we will say that $J$ is not random. Otherwise, we will leave it as is as an instance to $\operatorname{CSP}_{n^{r},\left(r, n^{-\frac{r}{4}}\right)}^{\mathrm{rand}, 1-\eta}\left(\mathrm{MXOR}_{q, K}, \neg \mathrm{MXOR}_{q, K}\right)$. To see that this reduction works, note that

- If $\operatorname{VAL}_{\mathrm{MXOR}}(J) \geq \eta$, we will either say that it is not random or produce an $\left(r, n^{-\frac{r}{4}}\right)$-pseudorandom instance with value $\geq \eta$.

\footnotetext{
${ }^{5}$ To be precise, the $(q, K)$-tuples and the labels are chosen uniformly, and independently from one another.

${ }^{6}$ To be precise, $J$ is chosen uniformly at random from all $(t, \tau)$-pseudo-random labeled $(q, K)$-formulas.
} 
- If $J$ is random, by lemma A.2, it is $\left(r, n^{-\frac{r}{4}}\right)$-pseudo-random with probability at least

$$
1-(2 n)^{K} 2 \exp \left(-2 n^{r} n^{-\frac{r}{2}}\right)=1-2 \exp \left(K \log (2 n)-2 n^{r} n^{-\frac{r}{2}}\right) \geq 1-o_{n}(1) .
$$

Hence, w.p. $\geq 1-o_{n}(1)$ the reduction will produce an instance. Now, conditioning on this event, the produced formulas is a random labeled $(q, K)$-formula that is $\left(r, n^{-\frac{r}{4}}\right)$-pseudorandom.

\section{Step IV: From MXOR to polynomials}

Let $\mathrm{POL}_{u, d}$ be the hypothesis class of all functions $h:\{0,-1,1\}^{u} \rightarrow\{ \pm\}$ that are thresholds of degree $\leq d$ polynomials. For $u=u(n), d=d(n), m=m(n)$ and $\eta=\eta(n)$ we consider the problem $\operatorname{POL}(u, d)_{m}^{\mathrm{s}-\text { scat }, \eta}$ of distinguishing a strongly-scattered sample from a sample with $\operatorname{Err}_{\mathrm{POL}_{d}}(S) \leq \eta$. Concretely, the input is a sample $S=\left\{\left(x_{1}, y_{1}\right), \ldots,\left(x_{m}, y_{m}\right)\right\} \subset\{-1,1,0\}^{u} \times\{ \pm 1\}$, and we say that the problem is easy if there exists an efficient randomized algorithm, $\mathcal{A}$ with the following properties. Its input is such a sample $S$, and its output satisfies:

- If $\operatorname{Err}_{\mathrm{POL}_{d}}(S) \leq \eta$, then

$$
\operatorname{Pr}_{\text {coins of } \mathcal{A}}(\mathcal{A}(S)=\text { "almost-realizable" }) \geq \frac{3}{4}
$$

- If $S$ is strongly scattered then, with probability $1-o_{n}(1)$ over the choice of the labels,

$$
\operatorname{Pr}_{\text {coins of } \mathcal{A}}\left(\mathcal{A}(J)=\text { "scattered") } \geq \frac{3}{4}\right. \text {. }
$$

Lemma A.11 For $d$ such that $\frac{K 2^{d} n^{d} \tau}{d}<d \leq t, \operatorname{CSP}_{m,(t, \tau)}^{\mathrm{rand}, 1-\eta}\left(\mathrm{MXOR}_{q, K}, \neg \mathrm{MXOR}_{q, K}\right)$ can efficiently reduced to $\operatorname{POL}(n q K, d)_{m}^{\mathrm{s}-\mathrm{scat}, \eta^{\prime}}$ where

$$
\eta^{\prime}=\eta+2 q \exp \left(-D\left(\frac{1}{2}+\frac{d}{2 K}, \frac{1}{2}+\frac{2^{d-1} n^{d} \tau}{d}\right) K\right)
$$

Proof Given a labeled $(q, K)$-formula $J=\left\{\left(C_{1}, y_{1}\right), \ldots,\left(C_{m}, y_{m}\right)\right\} \subset \mathcal{X}_{n, q, K} \times\{ \pm 1\}$ we will simply produce the sample $S=\left\{\left(\pi\left(C_{1}\right), y_{1}\right), \ldots,\left(\pi\left(C_{m}\right), y_{m}\right)\right\} \subset\{-1,1,0\}^{n q K} \times\{ \pm 1\}$. Here, $\Pi: \mathcal{X}_{n, q, K} \rightarrow$ $\{-1,1,0\}^{n q K}$ is the mapping $\pi\left(C^{1}, \ldots, C^{q}\right)=\left(\pi\left(C^{1}\right), \ldots, \pi\left(C^{q}\right)\right)$, where $\pi: \mathcal{X}_{n, K} \rightarrow\{-1,1,0\}^{n K}$ is as defined in section A.2.

Clearly if $J$ is random then $S$ is scattered. It remains to show that if $\operatorname{VAL}_{\mathrm{MxOR}}(J) \geq 1-\eta$ and $J$ is $(t, \tau)$-pseudo-random then there is a degree $\leq d$ polynomial that errs on $\leq \eta^{\prime}$ fraction of the examples.

Indeed, let $\psi \in\{ \pm 1\}^{n}$ be an assignment that satisfies $\geq 1-\eta$ fraction of $J^{\prime}$ th $(q, K)$-tuples. By lemma A.6, there is a polynomial $p:\{-1,1,0\}^{n K} \rightarrow \mathbb{R}$ of degree $\leq d$ that satisfies $p(\pi(C))=$ $\mathrm{XOR}(C(\psi))$ on $1-2 \exp \left(-D\left(\frac{1}{2}+\frac{d}{2 K}, \frac{1}{2}+\frac{2^{d-1} n^{d} \tau}{d}\right) K\right)$ fraction of $J$ 's $K$-tuples (here, $J$ 's $K$ tuples are the $K$-tuples that appear in one of $J$ 's $(q, K)$-tuples).

Let $p^{\prime}:\{-1,1,0\}^{n q K} \rightarrow \mathbb{R}$ be the degree $\leq d$ polynomial $p^{\prime}(x)=\sum_{j=1}^{q} p\left(z_{(j-1) K+1}, \ldots, z_{j K}\right)$. It is not hard to check that $\operatorname{sign}\left(p^{\prime}(\pi(C))\right)=\operatorname{MXOR}(C(\psi))$ on $1-2 q \exp \left(-D\left(\frac{1}{2}+\frac{d}{2 K}, \frac{1}{2}+\frac{2^{d-1} n^{d} \tau}{d}\right) K\right)$ fraction of $J$ 's $(q, K)$-tuples.

Therefore, we have that $\operatorname{sign}\left(p^{\prime}\left(\pi\left(C_{i}\right)\right)\right) \neq y_{i}$ for at most $\eta+2 q \exp \left(-D\left(\frac{1}{2}+\frac{d}{2 K}, \frac{1}{2}+\frac{2^{d-1} n^{d} \tau}{d}\right) K\right)$ fraction of the samples in $S$. 


\section{Step V: From polynomials to halfspaces}

Let $u=u(n), m=m(n)$ and $\eta=\eta(n)$. Similarly to $\operatorname{POL}(u, d)_{m}^{\mathrm{s}-\text { scat }, \eta}$, we define $\operatorname{HALF}(u)_{m}^{\mathrm{s}-\mathrm{scat}, \eta}$ as the problem of distinguishing a strongly-scattered sample consisting of $m$ examples in $\{ \pm 1\}^{u} \times\{ \pm 1\}$, from a sample with $\operatorname{Err}_{\mathrm{HALF}}(S) \leq \eta$.

Lemma A.12 The problem $\operatorname{POL}(u, d)_{m}^{\mathrm{s}-\mathrm{scat}, \eta}$ can efficiently reduced to $\operatorname{HALF}\left(2(u+1)^{d}\right)_{m}^{\mathrm{s}-\mathrm{scat}, \eta}$

Proof It will be convenient to decompose the reduction into two steps, where the second only deals with the issue of replacing $\{-1,1,0\}^{(u+1)^{d}}$ by $\{ \pm 1\}^{2(u+1)^{d}}$. Given a sample

$$
S=\left\{\left(x_{1}, y_{1}\right) \ldots,\left(x_{m}, y_{m}\right)\right\} \subset\{-1,1,0\}^{u} \times\{ \pm 1\}
$$

the reduction will first produce the sample

$$
\rho(S)=\left\{\left(\rho\left(x_{1}\right), y_{1}\right) \ldots,\left(\rho\left(x_{m}\right), y_{m}\right)\right\} \subset\{-1,1,0\}^{(u+1)^{d}} \times\{ \pm 1\},
$$

where $\rho:\{-1,1,0\}^{u} \rightarrow\{-1,1,0\}^{(u+1)^{d}}$ is defined as follows. We index the coordinates in $\{-1,1,0\}^{(u+1)^{d}}$ by the functions in $([u] \cup\{*\})^{[d]}$ and we let

$$
\forall f \in([u] \cup\{*\})^{[d]}, \quad \rho_{f}(x)=\Pi_{j=1}^{d} x_{f(j)} .
$$

(where $x_{*}:=1$ ). It is not hard to see that the collection of degree $\leq d$ polynomial functions from $\{-1,1,0\}^{u}$ to $\mathbb{R}$ equals to

$$
\left\{x \mapsto\langle w, \rho(x)\rangle \mid w \in \mathbb{R}^{(u+1)^{d}}\right\} .
$$

Hence, $\operatorname{Err}_{\mathrm{POL}_{u, d}}(S)=\operatorname{Err}_{\mathrm{HALF}}(\rho(S))$.

In the second step the reduction will produce the sample

$$
\Psi(\rho(S))=\left\{\left(\Psi\left(\rho\left(x_{1}\right)\right), y_{1}\right) \ldots,\left(\Psi\left(\rho\left(x_{m}\right)\right), y_{m}\right)\right\} \subset\{ \pm 1\}^{2(u+1)^{d}} \times\{ \pm 1\}
$$

$\Psi:\{-1,1,0\}^{(u+1)^{d}} \rightarrow\{ \pm 1\}^{2(u+1)^{d}}$ that is defined as follows:

$$
\Psi(x)=\left(\Psi\left(x_{1}\right), \ldots, \Psi\left(x_{n}\right)\right),
$$

where for $x \in\{0,-1,1\}, \Psi(x)=\left\{\begin{array}{ll}(1,1) & x=1 \\ (-1,-1) & x=-1 . \\ (-1,1) & x=0\end{array}\right.$ It is not hard to see that for every $w \in$ $\mathbb{R}^{(u+1)^{d}}$ we have $h_{w}=h_{w^{\prime}} \circ \Psi$ where $w^{\prime}=\frac{1}{2}\left(w_{1}, w_{1}, \ldots, w_{(u+1)^{d}}, w_{(u+1)^{d}}\right)$. Therefore we have

$$
\operatorname{Err}_{\text {HALF }}(\Psi(\rho(S))) \leq \operatorname{Err}_{\mathrm{HALF}}(\rho(S))=\operatorname{Err}_{\mathrm{POL}_{u, d}}(S)
$$

Also, it is clear that if $S$ is strongly scattered then so is $\Psi(\rho(S))$. To summarize, the reduction $S \mapsto \Psi(\rho(S))$ forms a reduction from $\operatorname{POL}(u, d)_{m}^{\mathrm{s}-\mathrm{scat}, \eta}$ to $\operatorname{HALF}\left(2(u+1)^{d}\right)_{m}^{\mathrm{s}-}$

\section{Connecting the dots}

We start with the hard problem $\operatorname{CSP}_{n^{r}}^{\text {rand, } \eta}\left(\mathrm{XOR}_{K}\right)$. Using Lemma A.7 and Lemma A.9, we reduce it to $\mathrm{CSP}_{\left\lfloor\frac{n^{r}}{q}\right\rfloor}^{\mathrm{rand}, 2^{-K}}\left(\mathrm{MXOR}_{q, K}, \neg \mathrm{MXOR}_{q, K}\right)$. Since $\eta$ is bounded away from $\frac{1}{2}$, this can be done with $q=C \cdot K$ for a constant $C=C(\eta)$. We can reduce farther to $\mathrm{CSP}_{n^{r-1}}^{\mathrm{rand}, 2^{-K}}\left(\mathrm{MXOR}_{q, K}, \neg \mathrm{MXOR}_{q, K}\right)$ (by simply throwing away random $\left\lfloor\frac{n^{r}}{q}\right\rfloor-n^{r-1}$ tuples from the input formula) 
Now, we use Lemma A.10 to reduce to $\operatorname{CSP}_{n^{r-1},\left(r-1, n^{-\frac{r-1}{4}}\right)}^{\text {rand, } 2^{-K}}\left(\operatorname{MXOR}_{q, K}, \neg \mathrm{MXOR}_{q, K}\right)$. Using Lemma A.11, for every $d$ such that $\frac{K 2^{d} n^{d-r+1}}{d}<d \leq r$ we can reduce to $\operatorname{POL}\left(n C K^{2}, d\right)_{n^{r-1}}^{\mathrm{s}-\mathrm{scat}, \eta^{\prime}}$ for

$$
\eta^{\prime} \leq 2^{-K}+2 C K \exp \left(-2\left(\frac{d}{2 K}-\frac{2^{d-1} n^{d-r+1}}{d}\right)^{2} K\right) .
$$

We will choose $d$ such that $d=o(r)$ and $d=\omega(\sqrt{\log (K) K})$ (the exact choice depend on the assumption we start with and will be specified later). It is not hard to check that for such a choice, it holds that for large enough $r$ and $K, \frac{K 2^{d} n^{d-r+1}}{d}<d \leq r-1$ and $\eta^{\prime} \leq 2^{-C_{1} \frac{d^{2}}{K}}$ for a constant $C_{1}>0$.

Now, by Lemma A.12 we can reduce farther to $\operatorname{HALF}\left(n^{4 d}\right)_{n^{r-1}}^{\mathrm{s}-\text { scat, } \eta^{\prime}}$. Putting $n^{\prime}=n^{4 d}$, we conclude that $\operatorname{HALF}\left(n^{\prime}\right)_{n^{\prime} \frac{\mathrm{s}-1}{4 d}}^{\mathrm{s} \text {-scat, } \eta^{\prime}}$. Since $d=o(r)$, this can be reduced farther (by simply randomly throwing examples from the input sample) to $\operatorname{HALF}\left(n^{\prime}\right)_{n^{\prime} a}^{\mathrm{s}-\mathrm{scat}, \eta^{\prime}}$ for any constant $a>0$. By Theorem 2.1, we conclude that there is no efficient learning algorithm that can return a hypothesis with nontrivial error on a distribution $\mathcal{D}$ on $\{ \pm 1\}^{n^{\prime}} \times\{ \pm 1\}$ that is $\eta^{\prime}$-almost realizable by halfspaces.

For the choice of $d$ and the calculation of $\eta^{\prime}$ in terms of $n^{\prime}$, we split to two cases, according to the assumption we started with.

Case 1 (Assumption 1.1). Here, $r=c \log (K) \sqrt{K}$ and $K$ is constant. We will choose $d=\log ^{\frac{2}{3}}(K) \sqrt{K}$. We will have $\eta^{\prime} \leq 2^{-\Omega\left(\log ^{\frac{4}{3}}(K)\right)}$. Since $K$ can be arbitrarily large, $\eta^{\prime}$ can be arbitrarily small.

Case 2 (Assumption 1.2). Here, $r=c K$ and $K=\log ^{s}(n)$. Here, we will choose $d=\frac{K}{\log \log K}$. Note that

$$
\begin{aligned}
\eta^{\prime} & \leq 2^{-C_{3} \frac{K}{(\log \log K)^{2}}} \\
& =2^{-C_{3} \frac{\log s(n)}{(\log \log K)^{2}}} \\
& \leq 2^{-\log ^{s-1}(n)}
\end{aligned}
$$

Now, $\log \left(n^{\prime}\right)=4 d \log (n)=\frac{4}{\log \log K} \log ^{s+1}(n) \leq \log ^{s+1}(n)$. Hence,

$$
\eta^{\prime} \leq 2^{-\log ^{\frac{s-1}{s+1}}\left(n^{\prime}\right)}
$$

Since $s$ can be arbitrarily large, we can get $\eta^{\prime} \leq 2^{-\log ^{1-\nu}\left(n^{\prime}\right)}$

\section{A.4 How to prove Theorems 1.4 and $1.5 ?$}

We next briefly explain how our argument can be extended to prove Theorems 1.4 and 1.5.

Theorem 1.4 is proved analogously to Theorem 1.3. The only difference is that we have to verify certain properties of the vector defining the halfspace that almost realizes the sample. Namely, we have to make sure that (i) the sum of its coefficients of is polynomial in the dimension and (ii) that whenever we guarantee that its prediction is correct, its inner product with the instance is $\geq 1$ in absolute value. These two facts can be straight forwardly verified, by carefully going over the proof.

Theorem 1.5 can also be proved analogously to Theorem 1.3. The only difference is that we have to verify that (i) the problem we start with cannot be solved efficiently using statistical queries and that (ii) the reduction steps can be done using statistical queries. This strategy can indeed 
be carried out, if one is using the result [39] of Feldman, Perkins and Vempala, that shows the SQ-hardness of the initial problem. However, a simpler strategy can be applied. Concretely, we can use our argument to reduce the uniform $K$-sparse-parity learning problem to the problems of learning halfspaces. In this parity problem, the learning algorithm is given an access to examples $(x, h(x))$ where $x \in\{ \pm 1\}^{n}$ is uniformly distributed and $h$ computes the XOR of $K$ unknown variables. It is known $[22,21]$ that no SQ-algorithm for the problem can return a classifier with error $\leq \frac{1}{2}-n^{-o(K)}$ using $n^{o(K)}$ queries with error parameters $n^{-o(K)}$. Lemma A.5 shows that for any XOR of $K$ variables, there is a polynomial threshold function of degree $d$ that agree with $h$ on all but $2 \exp \left(-\frac{d^{2}}{2 K}\right)$-fraction of the examples (w.r.t. the uniform distribution). As in the proof of Theorem 1.3, this fact establishes a reduction to the problem of agnostically learning halfspaces. All is left to show is that this reduction can be implemented using statistical queries.

Indeed, the reduction is of the following form. It introduces a mapping $\Psi:\{ \pm 1\}^{n} \rightarrow\{ \pm 1\}^{n^{r}}$ and reduce the original learning problem to the problem of learning $(\Psi(x), h(x))$, where $x$ is sampled from the original distribution (the uniform distribution in our case). Now, given a statistical query $Q:\{ \pm 1\}^{n^{r}} \times\{ \pm 1\} \rightarrow\{ \pm 1\}$, in order to evaluate $E_{x}[Q(\Psi(x), h(x))]$, we can simply query the oracle of the original problem with the function $\tilde{Q}(x, y):=Q(\Psi(x), y)$. 